ANALYSIS \& PDE

Volume $4 \quad$ No. $1 \quad 2011$ MARIUS PAICU AND ZHIFEI ZHANG
EGULARITY FOR THE NAVIER-STOKES EQUATIONS MARIUS PAICU AND ZHIFEI ZHANG
GLOBAL REGULARITY FOR THE NAVIER-STOKES EQUATIONS WITH SOME CLASSES OF LARGE INITIAL DATA 


\title{
GLOBAL REGULARITY FOR THE NAVIER-STOKES EQUATIONS WITH SOME CLASSES OF LARGE INITIAL DATA
}

\author{
MARIUS PAICU AND ZHIFEI ZHANG
}

\begin{abstract}
Chemin, Gallagher, and Paicu obtained in 2010 a class of large initial data that generate a global smooth solution to the three-dimensional, incompressible Navier-Stokes equation. The data varies slowly in the vertical direction - it is expressed as a function of $\varepsilon x_{3}$ - and it has a norm that blows up as the small parameter goes to zero. This type of initial data can be regarded as an ill prepared case, in contrast with the well prepared case treated in earlier papers. The data was supposed to evolve in a special domain, namely $\Omega=T_{h}^{2} \times \mathbb{R}_{v}$. The choice of a periodic domain in the horizontal variable played an important role.

The aim of this article is to study the case where the fluid evolves in the whole space $\mathbb{R}^{3}$. In this case, we have to overcome the difficulties coming from very low horizontal frequencies. We consider in this paper an intermediate situation between the well prepared case and ill prepared situation (the norms of the horizontal components of initial data are small but the norm of the vertical component blows up as the small parameter goes to zero). The proof uses the analytical-type estimates and the special structure of the nonlinear term of the equation.
\end{abstract}

\section{Introduction}

We study in this paper the Navier-Stokes equations with initial data which is slowly varying in the vertical variable. More precisely we consider the system

$$
\left\{\begin{array}{l}
\partial_{t} u+u \cdot \nabla u-\Delta u=-\nabla p \quad \text { in } \mathbb{R}_{+} \times \Omega, \\
\operatorname{div} u=0, \\
\left.u\right|_{t=0}=u_{0, \varepsilon},
\end{array}\right.
$$

where $\Omega=\mathbb{R}^{3}$ and $u_{0, \varepsilon}$ is a divergence-free vector field, whose dependence on the vertical variable $x_{3}$ will be chosen to be slow, meaning that it depends on $\varepsilon x_{3}$, where $\varepsilon$ is a small parameter. The goal is to prove the global existence in time of the solution generated by this type of initial data, with no smallness assumption on its norm.

This type of initial data (slowly varying in the vertical direction) has also been studied by Chemin, Gallagher, and coworkers. The case of well prepared initial data, of the form $\left(\varepsilon u_{0}^{h}\left(x_{h}, \varepsilon x_{3}\right), u_{0}^{3}\left(x_{h}, \varepsilon x_{3}\right)\right)$, was dealt with in [Chemin and Gallagher 2010]; the more difficult case of ill prepared initial data, of the form $\left(u^{h}\left(x_{h}, \varepsilon x_{3}\right), \varepsilon^{-1} u^{3}\left(x_{h}, \varepsilon x_{3}\right)\right)$, in [Chemin et al. 2011].

Here we consider a class of large initial data lying between those two cases, having the form

$$
u_{0, \varepsilon}=\left(\varepsilon^{\frac{1}{2}} u_{0}^{h}\left(x_{h}, \varepsilon x_{3}\right), \varepsilon^{-\frac{1}{2}} u_{0}^{3}\left(x_{h}, \varepsilon x_{3}\right)\right) .
$$

Z. Zhang is supported by NSF of China under Grant 10990013, 11071007, and SRF for ROCS, SEM. MSC2000: 35B65, 35Q35, 76D99, 76N10.

Keywords: Navier-Stokes equations, global well-posedness, large data. 
We recall some classical facts about the Navier-Stokes system, focusing on conditions that imply the global existence of the strong solution.

The first important result about the classical Navier-Stokes system [Leray 1934] asserted that for any initial data of finite energy there exists at least one global in time weak solution that satisfies the energy estimate. This solution is unique in $\mathbb{R}^{2}$, but it is not known to be unique in $\mathbb{R}^{3}$. Leray's result uses the structure of the nonlinear terms in order to obtain the energy inequality. The question of regularity of the weak solutions also remains open.

The Fujita-Kato theorem [1964] gives a partial answer for the construction of global unique solution: it allows one to construct a unique local in time solution in the homogeneous Sobolev spaces $\dot{H}^{\frac{1}{2}}\left(\mathbb{R}^{3}\right)$, or in the Lebesgue space $L^{3}\left(\mathbb{R}^{3}\right)$ [Kato 1984]. If the initial data is small compared to the viscosity, that is, if $\left\|u_{0}\right\|_{\dot{H}^{\frac{1}{2}}} \leq c v$, the strong solution exists globally in time. This result was generalized by Cannone, Meyer and Planchon [Cannone et al. 1994] to Besov spaces of negative index of regularity. Those authors proved that, if the initial data belongs to the Besov space $B_{p, \infty}^{-1+3 / p}\left(\mathbb{R}^{3}\right)$ and is small in the norm of this Besov space, compared to the viscosity, then the solution is global in time.

Later, Koch and Tataru [2001] obtained a unique global in time solution for the Navier-Stokes equation for small data belonging to a more general space of initial data, namely derivatives of BMO functions.

Concerning the methods for obtaining such results, we recall that the existence of a unique, global in time solution to the Navier-Stokes equation is a standard consequence of the Banach fixed point theorem, as long as the initial data is chosen small enough in some scale-invariant space (with norm invariant under the scaling $\left.\lambda u\left(\lambda^{2} t, \lambda x\right)\right)$ embedded in $\dot{B}_{\infty, \infty}^{-1}$ (the Besov space), with norm

$$
\|f\|_{\dot{B}_{\infty, \infty}^{-1}} \stackrel{\text { def }}{=} \sup _{t>0} t^{\frac{1}{2}}\left\|e^{t \Delta} f\right\|_{L^{\infty}} .
$$

See [Cannone et al. 1994; Fujita and Kato 1964; Koch and Tataru 2001; Weissler 1980] for proofs in various scale-invariant function spaces.

These theorems are general results of global existence for small initial data and do not take into account any algebraical properties of the nonlinear terms in the Navier-Stokes equations. Proving such results without any smallness assumption or geometrical invariance hypothesis implying conservation of quantities beyond the scaling is a challenge. Only modest progress has been made in that direction: see [Chemin et al. 2000; 2006; 2011; Chemin and Gallagher 2006; 2009; 2010; Chemin and Zhang 2007; Gallagher et al. 2003; Makhalov and Nikolaenko 2003; Raugel and Sell 1993] and references therein.

Here are some cases where large initial data is known to imply global existence of the solution:

For regular axisymmetric initial data without swirl, the Navier-Stokes system has a unique global in time solution. This result from [Ukhovskii and Iudovich 1968] is based on the conservation of some quantities beyond the scaling regularity level.

The case of large (in some sense) initial data for fluids evolving in thin domains was first considered in [Raugel and Sell 1993]. Roughly speaking, the three-dimensional Navier-Stokes system can be seen as a perturbation of the two-dimensional Navier-Stokes system if the vertical thickness of the domain is small enough. More generally, a solution exists globally in time if the initial data can be split as $v_{0}+w_{0}$, where $v_{0}$ is a two-dimensional divergence-free vector field in $L^{2}\left(T_{h}^{2}\right)$ and $w_{0} \in \dot{H}^{\frac{1}{2}}\left(T^{3}\right)$ satisfies

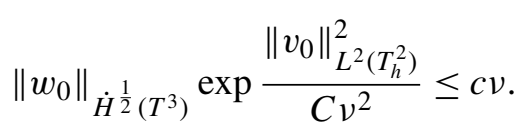


The case of initial data with large vortex in the vertical direction $\left(\operatorname{rot} u_{0}^{\varepsilon}=\operatorname{rot} u_{0}+\varepsilon^{-1}(0,0,1)\right.$ ), or equivalently the case of rotating fluids, was studied in [Makhalov and Nikolaenko 2003] for periodic domains and in [Chemin et al. 2000; Chemin et al. 2006] for a rotating fluid in $\mathbb{R}^{3}$ or in $\mathbb{R}^{2} \times(0,1)$. When the rotation is fast enough the fluid tends to have a two-dimensional behavior far from the boundary of the domain, by the Taylor-Proudman column theorem [Pedlovsky 1979]. For example, when the domain is $\mathbb{R}^{3}$ the fluctuation of this motion is dispersed to infinity and some Strichatz quantities became small [Chemin et al. 2000], which allow to obtain the global existence of the solution (for $\varepsilon$ small enough).

An important issue for the Navier-Stokes equations is to make the best possible use of the algebraical structure of the nonlinear terms. Some results have made crucial use of this structure, and have proved very fruitful.

The case of Navier-Stokes equations with vanishing vertical viscosity was first studied in [Chemin et al. 2000], which contains proofs of local existence for large data in anisotropic Sobolev spaces $H^{0, s}$, with $s>\frac{1}{2}$, and of global existence and uniqueness for small initial data. One of the key observations there is that, even if there is no vertical viscosity and thus no smoothing in the vertical variable, the partial derivative $\partial_{3}$ is only applied to the component $u_{3}$ in the nonlinear term. The divergence-free condition implies that $\partial_{3} u_{3}$ is regular enough to get good estimates of the nonlinear term.

Global existence of the solution for the anisotropic Navier-Stokes system with high oscillatory initial data was obtained in [Chemin and Zhang 2007].

A different idea, still using the special structure of the Navier-Stokes equation, was used in [Chemin and Gallagher 2006] to construct the first example of periodic initial data which is big in $C^{-1}$, and strongly oscillating in one direction which generate a global solution. The initial data is given by

$$
u_{0}^{N}=\left(N u_{h}\left(x_{h}\right) \cos \left(N x_{3}\right),-\operatorname{div}_{h} u_{h}\left(x_{h}\right) \sin \left(N x_{3}\right)\right),
$$

where $\left\|u_{h}\right\|_{L^{2}\left(T_{h}^{2}\right)} \leq C(\ln N)^{\frac{1}{9}}$. This result was generalized to $\mathbb{R}^{3}$ in [Chemin and Gallagher 2009].

The same authors [Chemin and Gallagher 2010] studied the Navier-Stokes equations for initial data that varies slowly in the vertical direction in the well prepared case. The well prepared case means that the norm of the initial data is large but does not blow up when the parameter $\varepsilon$ converges to zero. We note that important remarks on the pressure term and the bilinear term were used in this paper in order to obtain the global existence for large data.

The case of slowly varying initial data in the vertical direction (ell prepared initial data) was recently studied in [Chemin et al. 2011]. Here the horizontal components have large norm and the vertical component has a norm that blows up when the parameter goes to zero. After a change of scale, one obtains a Navier-Stokes type system with anisotropic viscosity $-v \Delta_{\varepsilon} u$, where $\Delta_{\varepsilon}=\Delta_{h}+\varepsilon^{2} \partial_{3}^{2}$, and anisotropic pressure gradient, namely $-\left(\nabla_{h} p, \varepsilon^{2} \partial_{3} p\right)$. In this equation there is a loss of regularity in the vertical variable in Sobolev estimates.

To overcome this difficulty one needs to work with analytical initial data. The most important tool was developed in [Chemin 2004] and consists in making analytical type estimates, and at the same time to control the size of the analyticity band. This is done by controlling nonlinear quantities that depend on the solution itself. Even in this situation, it is important to take into account very carefully the special structure of the Navier-Stokes equations. In fact, a global in time Cauchy-Kowalewskaya type theorem was obtained in [Chemin et al. 2011]. (Some local in time results for Euler and Prandtl equation with analytic initial data can be found in [Sammartino and Caflisch 1998].) 
In [Chemin et al. 2011] the fluid is supposed to evolve in a special domain, $\Omega=T_{h}^{2} \times \mathbb{R}_{v}$. This choice of domain is justified by the pressure term: the pressure satisfies the elliptic equation $\Delta_{\varepsilon} p=\partial_{i} \partial_{j}\left(u^{i} u^{j}\right)$, and consequently, $\nabla_{h} p=\left(-\Delta_{\varepsilon}\right)^{-1} \nabla_{h} \partial_{i} \partial_{j}\left(u^{i} u^{j}\right)$. Because $\Delta_{\varepsilon}^{-1}$ converges to $\Delta_{h}^{-1}$, it is important to control the low horizontal frequencies, in contrast with the case of the periodic torus in the horizontal variable, where we have only zero horizontal frequency and high horizontal frequencies.

In this paper we investigate the case where the fluid evolves in the full space $\mathbb{R}^{3}$. In that situation, we are able to solve globally in time the equation (conveniently rescaled in $\varepsilon$ ) for small analytic-type initial data. To do this, we need to control the low horizontal frequencies very precisely. Note that we can construct functional spaces where the operator $\Delta_{h}^{-1} \nabla_{h}\left(a \nabla_{h} b\right)$ is bounded. However, we still need to impose on the initial data more regularity control on low horizontal frequencies; thus we make the assumption $u_{0}\left(\cdot, x_{3}\right) \in L^{2}\left(\mathbb{R}_{h}^{2}\right) \cap \dot{H}^{-\frac{1}{2}}\left(\mathbb{R}_{h}^{2}\right)$. In the vertical variable we need to impose analyticity of the data. The method of the proof follows closely the argument of [Chemin et al. 2011], but instead to use pointwise estimates on the Fourier variables, we write an equation with a regularizing term in the vertical variable and we use energy estimates on anisotropic Sobolev spaces of the form $H^{0, s}$ respectively $H^{-\frac{1}{2}, s}$.

Our main result in the case of the full space $\mathbb{R}^{3}$ is the following (for notation see the next section).

Theorem 1.1. Let $a>0$ and $\frac{1}{2}>\alpha>0$. There exist positive constants $\varepsilon_{0}$ and $\eta$ such that, for any divergence-free field $v_{0}$ satisfying

$$
\left\|v_{0}\right\|_{X} \frac{1}{2}-\alpha\left\|v_{0}\right\|_{X \frac{1}{2}+\alpha} \leq \eta, \quad\left\|v_{0}\right\|_{X^{s}} \stackrel{\text { def }}{=}\left(\left\|e^{a\left\langle D_{3}\right\rangle} v_{0}\right\|_{H^{0, s}}+a^{-\frac{1}{2}}\left\|e^{a\left\langle D_{3}\right\rangle} v_{0}\right\|_{H^{-\frac{1}{2}, s}}\right)
$$

and for any $\varepsilon \in\left(0, \varepsilon_{0}\right)$, the Navier-Stokes system (NS) with initial data

$$
u_{0}^{\varepsilon}=\left(\varepsilon^{\frac{1}{2}} v_{0}^{h}\left(x_{h}, \varepsilon x_{3}\right), \varepsilon^{-\frac{1}{2}} v_{0}^{3}\left(x_{h}, \varepsilon x_{3}\right)\right)
$$

has a global smooth solution on $\mathbb{R}^{3}$.

We emphasize that we obtain the global wellposedness under a smallness condition which is invariant by the scaling of the equation, and this is the main motivation of Theorem 1.1.

As mentioned, to prove the theorem we will first transform the system using the change of scale

$$
u^{\varepsilon}\left(t, x_{h}, x_{3}\right)=\left(\varepsilon^{\frac{1}{2}} v^{h}\left(t, x_{h}, \varepsilon x_{3}\right), \varepsilon^{-\frac{1}{2}} v^{3}\left(t, x_{h}, \varepsilon x_{3}\right)\right)
$$

into a system of Navier-Stokes type, with a vertical vanishing viscosity, that is the Laplacian operator became $-v \Delta_{h} v-\varepsilon^{2} v \partial_{3} v$ and a changed pressure term which became $-\left(\nabla_{h} p, \varepsilon^{2} \partial_{3} p\right)$.

Taking advantage of the fact that we're working in $\mathbb{R}^{3}$, we can also consider a different type of initial data, with larger amplitude but strongly oscillating in the horizontal variables:

$$
u_{0}^{\varepsilon}=\left(\varepsilon^{-\frac{1}{2}} v_{0}^{h}\left(\varepsilon^{-1} x_{h}, x_{3}\right), \varepsilon^{-\frac{3}{2}} v_{0}^{3}\left(\varepsilon^{-1} x_{h}, x_{3}\right)\right) .
$$

This type of initial data has $\dot{B}_{\infty, \infty}^{-1}$ norm of the same order as the initial data in the previous theorem. In order to solve the Navier-Stokes equations with this new type of initial data, we make a different change of scale,

$$
u^{\varepsilon}\left(t, x_{h}, x_{3}\right)=\left(\varepsilon^{-\frac{1}{2}} v^{h}\left(\varepsilon^{-2} t, \varepsilon^{-1} x_{h}, x_{3}\right), \varepsilon^{-\frac{3}{2}} v^{3}\left(\varepsilon^{-2} t, \varepsilon^{-1} x_{h}, x_{3}\right)\right)
$$

and we note that the rescaled system that we obtain is exactly the same as in the proof of Theorem 1.1. Consequently, we obtain: 
Theorem 1.2. Let $a>0$ and $\frac{1}{2}>\alpha>0$. There exist positive constants $\varepsilon_{0}$ and $\eta$ such that, for any divergence-free field $v_{0}$ satisfying

$$
\left\|v_{0}\right\|_{X^{\frac{1}{2}-\alpha}}\left\|v_{0}\right\|_{X^{\frac{1}{2}+\alpha}} \leq \eta
$$

and any $\varepsilon \in\left(0, \varepsilon_{0}\right)$, the Navier-Stokes system (NS) with initial data

$$
u_{0}^{\varepsilon}=\left(\varepsilon^{-\frac{1}{2}} v_{0}^{h}\left(\varepsilon^{-1} x_{h}, x_{3}\right), \varepsilon^{-\frac{3}{2}} v_{0}^{3}\left(\varepsilon^{-1} x_{h}, x_{3}\right)\right)
$$

has a global smooth solution on $\mathbb{R}^{3}$.

\section{A simplified model}

We start with an equation already studied in [Chemin et al. 2011], where a complete proof of global wellposedness is given. For completeness and because we are going to use the method of energy estimates, we sketch the proof related to the energy estimates. Consider the equation

$$
\partial_{t} u+\gamma u+a(D)\left(u^{2}\right)=0,
$$

where $a(D)$ is a Fourier multiplier of order one. For any function $f$ such that the following definition makes sense, we define $e^{\delta|D|} f=\mathscr{F}^{-1}\left(e^{\delta|\xi|} \hat{f}(\xi)\right)$, where $\hat{f}=\mathscr{F} f$ denotes the Fourier transform and $\mathscr{F}^{-1}$ denotes the inverse of Fourier transform. Then, if the initial data satisfies

$$
\left\|e^{\delta|D|} u_{0}\right\|_{H^{s}} \leq c \gamma \quad \text { with } \delta>0 \text { and } s>\frac{1}{2} d,
$$

we have a global solution in the same space. The idea of the method in [Chemin 2004; Chemin et al. 2011] is the following: we want to control certain analytical quantities on the solution, but we must prevent a decrease in the radius of analyticity of the solution. Introduce $\theta(t)$, representing the loss of analyticity. We set $\Phi(t, \xi)=(\delta-\lambda \theta(t))|\xi|$ and $u_{\Phi}=\mathscr{F}^{-1}\left(e^{\Phi(t, \xi)} \hat{u}(\xi)\right)$. The function $\theta(t)$ is defined as the unique solution of the ordinary differential equation

$$
\dot{\theta}(t)=\left\|u_{\Phi}\right\|_{H^{s}}, \quad \theta(0)=0 .
$$

The computations that follow are performed under the condition $\theta(t) \leq \delta / \lambda$ (which implies $\Phi \geq 0$ ). The equation satisfied by $\hat{u}_{\phi}$ is

$$
\partial_{t} \hat{u}_{\Phi}+\gamma \hat{u}_{\Phi}+\lambda \dot{\theta}(t)|\xi| \hat{u}_{\Phi}+a(\xi) e^{\Phi}\left(\widehat{u^{2}}\right)=0 .
$$

This contains an extra-regularizing term, since we control a quantity that takes into account the analyticity of the solution. As $\dot{\theta}$ approaches 0 , we obtain by an energy estimate in $H^{s}$ the inequality

$$
\frac{1}{2} \frac{d}{d t}\left\|u_{\Phi}\right\|_{H^{s}}^{2}+\gamma\left\|u_{\Phi}\right\|_{H^{s}}^{2}+\lambda \dot{\theta}(t)\left\||D|^{\frac{1}{2}} u_{\Phi}\right\|_{H^{s}}^{2} \leq C\left|\left(a(D)\left(u^{2}\right)_{\Phi}, u_{\Phi}\right)_{H^{s}}\right| .
$$

Following the proof of [Chemin et al. 2011, Lemma 2.1] (which uses the important fact that $e^{\Phi(t, \xi)}$ is a sublinear function, and also the classical Bony decomposition [1981]), we get

$$
\left.\mid a(D)\left(u^{2}\right)_{\Phi}, u_{\Phi}\right)\left|\leq C\left\|u_{\Phi}\right\|_{H^{s}}\left\||D|^{\frac{1}{2}} u_{\Phi}\right\|_{H^{s}}^{2} .\right.
$$

Choosing $\lambda=4 C$ we obtain

$$
\dot{\theta}(t)=\left\|u_{\Phi}(t)\right\|_{H^{s}} \leq 2\left\|e^{\delta|D|} u_{0}\right\|_{H^{s}} e^{-\gamma t},
$$


which, for $u_{0}$ small enough, gives

$$
\theta(t) \leq \gamma^{-1}\left\|e^{\delta|D|} u_{0}\right\|_{H^{s}} \leq \delta \lambda^{-1} .
$$

This allows us to obtain the global in time existence of the solution.

\section{Structure of the proof}

Reduction to a rescaled problem. We seek a solution of the form

$$
u_{\varepsilon}(t, x) \stackrel{\text { def }}{=}\left(\varepsilon^{\frac{1}{2}} v^{h}\left(t, x_{h}, \varepsilon x_{3}\right), \varepsilon^{-\frac{1}{2}} v^{3}\left(t, x_{h}, \varepsilon x_{3}\right)\right) .
$$

This leads to the rescaled Navier-Stokes system

$$
\left\{\begin{array}{l}
\partial_{t} v^{h}-\Delta_{h} v^{h}-\varepsilon^{2} \partial_{3}^{2} v^{h}+\varepsilon^{\frac{1}{2}} v \cdot \nabla v^{h}=-\nabla^{h} q \\
\partial_{t} v^{3}-\Delta_{h} v^{3}-\varepsilon^{2} \partial_{3}^{2} v^{3}+\varepsilon^{\frac{1}{2}} v \cdot \nabla v^{3}=-\varepsilon^{2} \partial_{3} q \\
\operatorname{div} v=0 \\
v(0)=v_{0}(x)
\end{array}\right.
$$

where $\Delta_{h} \stackrel{\text { def }}{=} \partial_{1}^{2}+\partial_{2}^{2}$ and $\nabla_{h} \stackrel{\text { def }}{=}\left(\partial_{1}, \partial_{2}\right)$. As there is no boundary, the rescaled pressure $q$ can be computed with the formula

$$
-\Delta_{\varepsilon} q=\varepsilon^{\frac{1}{2}} \operatorname{div}_{h}(v \cdot \nabla v), \quad \Delta_{\varepsilon}=\Delta_{h}+\varepsilon^{2} \partial_{3}^{2} .
$$

When $\varepsilon$ tends to zero, $\Delta_{\varepsilon}^{-1}$ looks like $\Delta_{h}^{-1}$. Thus, for low horizontal frequencies, an expression of $\nabla_{h} \Delta_{h}^{-1}$ cannot be estimated in $L^{2}$. This is one reason for working in $T_{h}^{2} \times \mathbb{R}_{v}$ in [Chemin et al. 2011]. To obtain a similar result in $\mathbb{R}^{3}$, we need to introduce the following anisotropic Sobolev space.

Definition 3.1. Let $s, \sigma \in \mathbb{R}, \sigma<1$. The anisotropic Sobolev space $H^{\sigma, s}$ is defined by

$$
H^{\sigma, s}=\left\{f \in \mathscr{Y}^{\prime}\left(\mathbb{R}^{3}\right):\|f\|_{H^{\sigma, s}}<\infty\right\},
$$

where

$$
\|f\|_{H^{\sigma, s}}^{2} \stackrel{\text { def }}{=} \int_{\mathbb{R}^{3}}\left|\xi_{h}\right|^{2 \sigma}\left(1+\left|\xi_{3}\right|^{2}\right)^{s}|\hat{f}(\xi)|^{2} d \xi, \quad \xi=\left(\xi_{h}, \xi_{3}\right) .
$$

For any $f, g \in H^{\sigma, s}$, we set

$$
(f, g)_{H^{\sigma, s}} \stackrel{\text { def }}{=}\left(\left|D_{h}\right|^{\sigma}\left\langle D_{3}\right\rangle^{s} f,\left|D_{h}\right|^{\sigma}\left\langle D_{3}\right\rangle^{s} g\right)_{L^{2}}, \quad\left\langle D_{3}\right\rangle=\left(1+\left|D_{3}\right|^{2}\right)^{\frac{1}{2}} .
$$

Theorem 3.2. Let $a>0, \frac{1}{2}>\alpha>0$. There exist two positive constants $\varepsilon_{0}$ and $\eta$ such that for any divergence-free fields $v_{0}$ satisfying

$$
\left\|v_{0}\right\|_{X \frac{1}{2}-\alpha}\left\|v_{0}\right\|_{X \frac{1}{2}+\alpha} \leq \eta,
$$

and for any $\varepsilon \in\left(0, \varepsilon_{0}\right),\left(\mathrm{RNS}_{\varepsilon}\right)$ has a global smooth solution on $\mathbb{R}^{3}$. 
Definition of the functional setting. As in [Chemin et al. 2011], the proof relies on exponential decay estimates for the Fourier transform of the solution. Thus, for any locally bounded function $\Psi$ on $\mathbb{R}^{+} \times \mathbb{R}^{3}$ and for any function $f$, continuous in time and compactly supported in Fourier space, we define

$$
f_{\Psi}(t) \stackrel{\text { def }}{=} \mathscr{F}^{-1}\left(e^{\Psi(t, \cdot)} \widehat{f}(t, \cdot)\right) .
$$

Now we introduce a key quantity we want to control in order to prove the theorem. We define the function $\theta(t)$ by

$$
\begin{aligned}
\dot{\theta}(t) \stackrel{\text { def }}{=} \frac{1}{a}\left(\varepsilon\left\|v_{\Phi}^{h}(t)\right\|_{H \frac{1}{2}, \frac{1}{2}-\alpha}\left\|v_{\Phi}^{h}(t)\right\|_{H \frac{1}{2}, \frac{1}{2}+\alpha}+\left\|v_{\Phi}^{3}(t)\right\|_{H \frac{1}{2}, \frac{1}{2}-\alpha}\left\|v_{\Phi}^{3}(t)\right\|_{H \frac{1}{2}, \frac{1}{2}+\alpha}\right) \\
+\left\|\nabla_{h} v_{\Phi}(t)\right\|_{H^{0, \frac{1}{2}-\alpha}\left\|\nabla_{h} v_{\Phi}(t)\right\|_{H^{0, \frac{1}{2}+\alpha}}}
\end{aligned}
$$

with $\theta(0)=0$, and we also define

$$
\Psi(t) \stackrel{\text { def }}{=}\left\|v_{\Phi}(t)\right\|_{H^{0, \frac{1}{2}-\alpha}}\left\|v_{\Phi}(t)\right\|_{H^{0, \frac{1}{2}+\alpha}}
$$

where

$$
\Phi(t, \xi) \stackrel{\text { def }}{=} a(1-\lambda \theta(t))\left\langle\xi_{3}\right\rangle,
$$

for some $\lambda$ that will be chosen later on. We denoted by $\left\langle\xi_{3}\right\rangle=\left(1+\left|\xi_{3}\right|^{2}\right)^{\frac{1}{2}}$ which is a sublinear function.

\section{Main steps of the proof.}

Proposition 3.3. Let $s>0$. A constant $C$ exists such that, for any positive $\lambda$ and for any $t$ satisfying $\theta(t) \leq 1 / \lambda$, we have

$$
\begin{aligned}
& \int_{0}^{t}\left(\left\|\varepsilon^{\frac{1}{2}} v_{\Phi}^{h}(\tau)\right\|_{H \frac{1}{2}, s}^{2}+\left\|v_{\Phi}^{3}(\tau)\right\|_{H \frac{1}{2}, s}^{2}\right) d \tau \\
& \leq \exp (C \theta(t))\left[\left\|e^{a\left\langle D_{3}\right\rangle} v_{0}\right\|_{H^{-\frac{1}{2}, s}}^{2}+C a \int_{0}^{t} \dot{\theta}(\tau)\left\|v_{\Phi}^{h}(\tau)\right\|_{H^{0, s}}^{2} d \tau\right. \\
& \left.\quad+C \int_{0}^{t} \Psi(\tau)\left(\left\|\varepsilon^{\frac{1}{2}} v_{\Phi}^{h}(\tau)\right\|_{H \frac{1}{2}, s}^{2}+\left\|v_{\Phi}^{3}(\tau)\right\|_{H \frac{1}{2}, s}^{2}\right) d \tau+\frac{a}{10} \int_{0}^{t}\left\|\nabla_{h} v_{\Phi}^{h}(\tau)\right\|_{H^{0, s}}^{2} d \tau\right] .
\end{aligned}
$$

Proposition 3.4. Let $1>s>0$. Then there exist $C$ and $\lambda_{0}$ such that for any $\lambda \geq \lambda_{0}$ and for any $t$ satisfying $\theta(t) \leq 1 / \lambda$, we have

$$
\begin{aligned}
\left\|v_{\Phi}(t)\right\|_{H^{0, s}}^{2}+ & \int_{0}^{t}\left\|\nabla_{h} v_{\Phi}(\tau)\right\|_{H^{0, s}}^{2} d \tau \\
& \leq \exp (C \theta(t))\left[\left\|e^{a\left\langle D_{3}\right\rangle} v_{0}\right\|_{H^{0, s}}^{2}+C \int_{0}^{t} \Psi(\tau)\left\|v_{\Phi}(\tau)\right\|_{H^{1, s}}^{2} d \tau+\frac{1}{10} \int_{0}^{t} \frac{1}{a}\left\|v_{\Phi}^{3}(\tau)\right\|_{H^{\frac{1}{2}, s}}^{2} d \tau\right] .
\end{aligned}
$$

Proposition 3.3 will be proved in Section 4, and Proposition 3.4 in Section 5. For the moment, let us assume that they are true and conclude the proof of Theorem 3.2. As in [Chemin et al. 2011], we use a continuation argument. For any $\lambda \geq \lambda_{0}$ and $\eta$, define

$$
\mathscr{T}_{\lambda} \stackrel{\text { def }}{=}\{T: \theta(T)+\Psi(T) \leq 4 \eta\}
$$


Similar to the argument in [Chemin et al. 2011], $\mathscr{T}_{\lambda}$ is of the form $\left[0, T^{*}\right)$ for some positive $T^{*}$. Thus, it suffices to prove that $T^{*}=+\infty$. In order to two propositions, we need to assume that $\theta(T) \leq 1 / \lambda$, which leads to the condition

$$
4 \eta \leq \frac{1}{\lambda}
$$

We set

$$
F_{S}(t) \stackrel{\text { def }}{=}\left\|v_{\Phi}(t)\right\|_{H^{0, s}}^{2}+\int_{0}^{t}\left\|\nabla_{h} v_{\Phi}(\tau)\right\|_{H^{0, s}}^{2} d \tau+\frac{1}{a} \int_{0}^{t}\left(\left\|\varepsilon^{\frac{1}{2}} v_{\Phi}^{h}(\tau)\right\|_{H \frac{1}{2}, s}^{2}+\left\|v_{\Phi}^{3}(\tau)\right\|_{H \frac{1}{2}, s}^{2}\right) d \tau .
$$

From Propositions 3.3 and 3.4, it follows that for all $T \in \mathscr{T}_{\lambda}$,

$$
\begin{aligned}
F_{S}(t) \leq \frac{10}{9} \exp (4 C \eta)\left(\frac{1}{a}\left\|e^{a\left\langle D_{3}\right\rangle} v_{0}\right\|_{H^{-\frac{1}{2}, s}}^{2}+\right. & \left\|e^{a\left\langle D_{3}\right\rangle} v_{0}\right\|_{H^{0, s}}^{2}+C \int_{0}^{t} \dot{\theta}(\tau)\left\|v_{\Phi}^{h}(\tau)\right\|_{H^{0, s}}^{2} d \tau \\
& \left.+4 C \eta \int_{0}^{t}\left(\frac{1}{a}\left\|\varepsilon^{\frac{1}{2}} v_{\Phi}^{h}(\tau)\right\|_{H^{\frac{1}{2}, s}}^{2}+\frac{1}{a}\left\|v_{\Phi}^{3}(\tau)\right\|_{H^{\frac{1}{2}, s}}^{2}+\left\|v_{\Phi}(\tau)\right\|_{H^{1, s}}^{2}\right) d \tau\right)
\end{aligned}
$$

Now we choose $\eta$ such that

$$
\exp (4 C \eta) \leq \frac{9}{8}, \quad 4 C \eta \exp (4 C \eta) \leq \frac{1}{4}
$$

With this choice of $\eta$, we infer from Gronwall's inequality that

$$
F_{s}(t)<2\left(\frac{1}{a}\left\|e^{a\left\langle D_{3}\right\rangle} v_{0}\right\|_{H^{-\frac{1}{2}, s}}^{2}+\left\|e^{a\left\langle D_{3}\right\rangle} v_{0}\right\|_{H^{0, s}}^{2}\right) \leq 2\left\|v_{0}\right\|_{X^{s}}^{2} .
$$

Taking $s=\frac{1}{2}-\alpha$ and $s=\frac{1}{2}+\alpha$ respectively, we obtain

$$
a^{-1} \theta(t)+\Psi(t) \leq 2 F_{\frac{2}{2}-\alpha}^{\frac{1}{2}}(t) F_{\frac{2}{2}+\alpha}^{\frac{1}{2}}(t)<4\left\|v_{0}\right\|_{X^{\frac{1}{2}-\alpha}}\left\|v_{0}\right\|_{X^{\frac{1}{2}+\alpha}} \leq 4 \eta,
$$

which ensures that $T^{*}=+\infty$, thus concluding the proof of Theorem 3.2.

\section{The action of subadditive phases on products}

For any function $f$, we denote by $f^{+}$the inverse Fourier transform of $|\hat{f}|$. Let us notice that the map $f \mapsto f^{+}$preserves the norm of all $H^{\sigma, s}$ spaces. Throughout this section, $\Psi$ will denote a locally bounded function on $\mathbb{R}^{+} \times \mathbb{R}^{3}$ which satisfies the following inequality

$$
\Psi(t, \xi) \leq \Psi(t, \xi-\eta)+\Psi(t, \eta)
$$

Before presenting the product estimates, let us recall the Littlewood-Paley decomposition. Choose two nonnegative even functions $\chi, \varphi \in \mathscr{S}(\mathbb{R})$ supported, respectively, in $\mathscr{B}=\left\{\xi \in \mathbb{R},|\xi| \leq \frac{4}{3}\right\}$ and $\mathscr{C}=\left\{\xi \in \mathbb{R}, \frac{3}{4} \leq|\xi| \leq \frac{8}{3}\right\}$ such that

$$
\begin{aligned}
& \chi(\xi)+\sum_{j \geq 0} \varphi\left(2^{-j} \xi\right)=1 \quad \text { for } \xi \in \mathbb{R} \\
& \sum_{j \in \mathbb{Z}} \varphi\left(2^{-j} \xi\right)=1 \quad \text { for } \xi \in \mathbb{R} \backslash\{0\} .
\end{aligned}
$$


The frequency localization operators $\Delta_{j}^{v}$ and $S_{j}^{v}$ in the vertical direction are defined by

$$
\begin{aligned}
& \Delta_{j}^{v} f= \begin{cases}\mathscr{F}^{-1}\left(\varphi\left(2^{-j}\left|\xi_{3}\right|\right) \hat{f}\right) & \text { for } j \geq 0, \\
S_{0}^{v} f & \text { for } j=-1, \\
0 & \text { for } j \leq-2,\end{cases} \\
& S_{j}^{v} f=\mathscr{F}^{-1}\left(\chi\left(2^{-j}\left|\xi_{3}\right|\right) \hat{f}\right)=\sum_{j^{\prime} \leq j-1} \Delta_{j^{\prime}}^{v} f .
\end{aligned}
$$

The frequency localization operators $\dot{\Delta}_{j}^{h}$ and $S_{j}^{h}$ in the horizontal direction are defined by

$$
\dot{\Delta}_{j}^{h} f=\mathscr{F}^{-1}\left(\varphi\left(2^{-j}\left|\xi_{h}\right|\right) \hat{f}\right), \quad S_{j}^{h} f=\sum_{j^{\prime} \leq j-1} \dot{\Delta}_{j^{\prime}}^{h} f \quad \text { for } j \in \mathbb{Z} .
$$

It is easy to verify that

$$
\|f\|_{H^{\sigma, s}}^{2} \approx \sum_{j, k \in \mathbb{Z}} 2^{2 j s} 2^{2 k \sigma}\left\|\Delta_{j}^{v} \dot{\Delta}_{k}^{h} f\right\|_{L^{2}}^{2}
$$

In the sequel, we will constantly use the Bony's decomposition [1981]:

$$
f g=T_{f}^{v} g+R_{f}^{v} g,
$$

with

$$
T_{f}^{v} g=\sum_{j} S_{j-1}^{v} f \Delta_{j}^{v} g, \quad R_{f}^{v} g=\sum_{j} S_{j+2}^{v} f \Delta_{j}^{v} g .
$$

We also use the Bony's decomposition in the horizontal direction:

$$
f g=T_{f}^{h} g+T_{f}^{h} g+R^{h}(f, g),
$$

with

$$
T_{f}^{h} g=\sum_{j} S_{j-1}^{h} f \dot{\Delta}_{j}^{h} g, \quad R^{h}(f, g)=\sum_{\left|j^{\prime}-j\right| \leq 1} \dot{\Delta}_{j}^{h} f \dot{\Delta}_{j^{\prime}}^{h} g .
$$

Lemma 4.1 (Bernstein's inequality). Let $1 \leq p \leq q \leq \infty$. If $f \in L^{p}\left(\mathbb{R}^{d}\right)$, there exists a constant $C$ independent of $f, j$ such that

$$
\begin{array}{ll}
\operatorname{supp} \hat{f} \subset\left\{|\xi| \leq C 2^{j}\right\} & \Longrightarrow\left\|\partial^{\alpha} f\right\|_{L^{q}} \leq C 2^{j|\alpha|+d j\left(\frac{1}{p}-\frac{1}{q}\right)}\|f\|_{L^{p}}, \\
\operatorname{supp} \hat{f} \subset\left\{\frac{1}{C} 2^{j} \leq|\xi| \leq C 2^{j}\right\} & \Longrightarrow\|f\|_{L^{p}} \leq C 2^{-j|\alpha|} \sup _{|\beta|=|\alpha|}\left\|\partial^{\beta} f\right\|_{L^{p}} .
\end{array}
$$

Lemma 4.2. Let $s>0, \sigma_{1}, \sigma_{2}<1$ such that $\sigma_{1}+\sigma_{2}>0$ and $\frac{1}{2}>\alpha>0$. Assume that $a_{\Psi} \in H^{\sigma_{1}, \frac{1}{2}+\alpha}$ and $b_{\Psi} \in H^{\sigma_{2}, s}$. Then

$$
\left\|\left[\Delta_{j}^{v} \dot{\Delta}_{k}^{h}\left(T_{a}^{v} b\right)\right]_{\Psi}\right\|_{L^{2}}+\left\|\left[\Delta_{j}^{v} \dot{\Delta}_{k}^{h}\left(R_{a}^{v} b\right)\right]_{\Psi}\right\|_{L^{2}} \leq C c_{j, k} 2^{\left(1-\sigma_{1}-\sigma_{2}\right) k} 2^{-j s}\left\|a_{\Psi}\right\|_{H^{\sigma_{1}, \frac{1}{2}-\alpha}}^{\frac{1}{2}}\left\|a_{\Psi}\right\|_{H^{\sigma_{1}, \frac{1}{2}+\alpha}}^{\frac{1}{2}}\left\|b_{\Psi}\right\|_{H^{\sigma_{2}, s}},
$$

with the sequence $\left(c_{j, k}\right)_{j, k \in \mathbb{Z}}$ of positive numbers satisfying

$$
\sum_{j, k} c_{j, k}^{2} \leq 1
$$


Proof. Let us firstly prove the case when the function $\Psi$ is identically 0 . Below we only present the proof of $R_{a}^{v} b$, the proof for $T_{a}^{v} b$ is very similar. Using Bony's decomposition (4-4) in the horizontal direction, we write

$$
\begin{aligned}
\Delta_{j} \dot{\Delta}_{k}^{h}\left(R_{a}^{v} b\right)=\sum_{j^{\prime}} \Delta_{j}^{v} \dot{\Delta}_{k}^{h}\left(S_{j^{\prime}+2}^{v} a \Delta_{j^{\prime}}^{v} b\right) & =\sum_{j^{\prime}} \Delta_{j}^{v} \dot{\Delta}_{k}^{h}\left(T_{S_{j^{\prime}+2}^{v} a}^{h} \Delta_{j^{\prime}}^{v} b+T_{\Delta_{j^{\prime}}^{v}}^{h} S_{j^{\prime}+2}^{v} a+R^{h}\left(S_{j^{\prime}+2}^{v} a, \Delta_{j^{\prime}}^{v}, b\right)\right) \\
& \stackrel{\text { def }}{=} \mathrm{I}+\mathrm{II}+\mathrm{III} .
\end{aligned}
$$

Considering the support of the Fourier transform of $T_{S_{j^{\prime}+2}^{v}}^{h} \Delta_{j^{\prime}}^{v} b$, we have

$$
\mathrm{I}=\sum_{j^{\prime} \geq j-4} \sum_{\left|k^{\prime}-k\right| \leq 4} \Delta_{j}^{v} \dot{\Delta}_{k}^{h}\left(S_{j^{\prime}+2}^{v} S_{k^{\prime}-1}^{h} a \Delta_{j^{\prime}}^{v} \dot{\Delta}_{k^{\prime}}^{h} b\right) \text {. }
$$

Then, by Lemma 4.1,

$$
\begin{aligned}
\|\mathrm{I}\|_{L^{2}} & \leq C \sum_{j^{\prime} \geq j-4} \sum_{\left|k^{\prime}-k\right| \leq 4}\left\|S_{j^{\prime}+2}^{v} S_{k^{\prime}-1}^{h} a \Delta_{j^{\prime}} \dot{\Delta}_{k^{\prime}}^{h} b\right\|_{L^{2}} \\
& \leq C \sum_{j^{\prime} \geq j-4} \sum_{\left|k^{\prime}-k\right| \leq 4}\left\|S_{j^{\prime}+2}^{v} S_{k^{\prime}-1}^{h} a\right\|_{L^{\infty}}\left\|\Delta_{j^{\prime}}^{v} \dot{\Delta}_{k^{\prime}}^{h} b\right\|_{L^{2} .}
\end{aligned}
$$

We use Lemma 4.1 again to get

$$
\begin{aligned}
& \left\|S_{j^{\prime}+2}^{v} S_{k^{\prime}-1}^{h} a\right\|_{L^{\infty}} \leq \sum_{j^{\prime \prime} \leq j^{\prime}+1} \sum_{k^{\prime \prime} \leq k^{\prime}-2}\left\|\Delta_{j^{\prime \prime}}^{v} \dot{\Delta}_{k^{\prime \prime}}^{h} a\right\|_{L^{\infty}} \leq C \sum_{j^{\prime \prime} \leq j^{\prime}+1} \sum_{k^{\prime \prime} \leq k^{\prime}-2} 2^{k^{\prime \prime}}\left\|\Delta_{j^{\prime \prime}}^{v} \dot{\Delta}_{k^{\prime \prime}}^{h} a\right\|_{L_{x_{3}}^{\infty} L_{x_{h}}^{2}} \\
& \leq C \sum_{j^{\prime \prime} \leq j^{\prime}+1} \sum_{k^{\prime \prime} \leq k^{\prime}-2} 2^{j^{\prime \prime} / 2} 2^{k^{\prime \prime}}\left\|\Delta_{j^{\prime \prime}}^{v} \dot{\Delta}_{k^{\prime \prime}}^{h} a\right\|_{L^{2}} \leq C 2^{\left(1-\sigma_{1}\right) k}\|a\|_{H^{\sigma_{1}, \frac{1}{2}-\alpha}}^{\frac{1}{2}}\|a\|_{H^{\sigma_{1}, \frac{1}{2}+\alpha}}^{\frac{1}{2}},
\end{aligned}
$$

from which it follows that

$$
\begin{aligned}
\|\mathrm{I}\|_{L^{2}} & \leq C 2^{\left(1-\sigma_{1}\right) k}\|a\|_{H^{\sigma_{1}, \frac{1}{2}-\alpha}}^{\frac{1}{2}}\|a\|_{H^{\sigma_{1}, \frac{1}{2}+\alpha}}^{\frac{1}{2}} \sum_{j^{\prime} \geq j-4} \sum_{\left|k^{\prime}-k\right| \leq 4}\left\|\Delta_{j^{\prime}}^{v} \dot{\Delta}_{k^{\prime}}^{h} b\right\|_{L^{2}} \\
& \leq C c_{j, k} 2^{-j s} 2^{\left(1-\sigma_{1}-\sigma_{2}\right) k}\|a\|_{H^{\sigma_{1}, \frac{1}{2}-\alpha}}^{\frac{1}{2}}\|a\|_{H^{\sigma_{1}, \frac{1}{2}+\alpha}}^{\frac{1}{2}}\|b\|_{H^{\sigma_{2}, s} .}
\end{aligned}
$$

Similarly, we have

$$
\mathrm{II}=\sum_{j^{\prime} \geq j-4} \sum_{\left|k^{\prime}-k\right| \leq 4} \Delta_{j}^{v} \dot{\Delta}_{k}^{h}\left(\Delta_{j^{\prime}}^{v} S_{k^{\prime}-1}^{h} b S_{j^{\prime}+2}^{v} \dot{\Delta}_{k^{\prime}}^{h} a\right)
$$

Then, by Lemma 4.1,

$$
\begin{aligned}
\|\mathrm{II}\|_{L^{2}} & \leq C \sum_{j^{\prime} \geq j-4\left|k^{\prime}-k\right| \leq 4}\left\|\Delta_{j^{\prime}}^{v} S_{k^{\prime}-1}^{h} b\right\|_{L_{x_{3}}^{2} L_{x_{h}}^{\infty}}\left\|S_{j^{\prime}+2}^{v} \dot{\Delta}_{k^{\prime}}^{h} a\right\|_{L_{x_{h}}^{2} L_{x_{3}}^{\infty}} \\
& \leq C 2^{-j s} 2^{\left(1-\sigma_{1}-\sigma_{2}\right) k}\|a\|_{H^{\sigma_{1}, \frac{1}{2}-\alpha}}^{\frac{1}{2}}\|a\|_{H^{\sigma_{1}, \frac{1}{2}+\alpha}}^{\frac{1}{2}}\|b\|_{H^{\sigma_{2}, s}} \sum_{j^{\prime} \geq j-4\left|k^{\prime}-k\right| \leq 4} \sum 2^{-\left(j^{\prime}-j\right) s} c_{k^{\prime}} c_{j^{\prime}} \\
& \leq C c_{j, k} 2^{-j s} 2^{\left(1-\sigma_{1}-\sigma_{2}\right) k}\|a\|_{H^{\sigma_{1}, \frac{1}{2}-\alpha}}^{\frac{1}{2}}\|a\|_{H^{\sigma_{1}, \frac{1}{2}+\alpha}}^{\frac{1}{2}}\|b\|_{H^{\sigma_{2}, s} .}
\end{aligned}
$$


We turn to III. We have

$$
\mathrm{III}=\sum_{j^{\prime} \geq j-4} \sum_{\substack{k^{\prime}, k^{\prime \prime} \geq k-2 \\\left|k^{\prime}-k^{\prime \prime}\right| \leq 1}} \Delta_{j}^{v} \dot{\Delta}_{k}^{h}\left(S_{j^{\prime}+2}^{v} \dot{\Delta}_{k^{\prime}}^{h} a \Delta_{j^{\prime}}^{v} \dot{\Delta}_{k^{\prime \prime}}^{h} b\right) .
$$

So, by Lemma 4.1,

$$
\begin{aligned}
\|\mathrm{III}\|_{L^{2}} & \leq C \sum_{j^{\prime} \geq j-4} \sum_{\substack{k^{\prime}, k^{\prime \prime} \geq k-2 \\
\left|k^{\prime}-k^{\prime \prime}\right| \leq 1}} 2^{k}\left\|S_{j^{\prime}+2}^{v} \dot{\Delta}_{k^{\prime}}^{h} a \Delta_{j^{\prime}}^{v} \dot{\Delta}_{k^{\prime \prime}}^{h} b\right\|_{L_{x_{3}}^{2} L_{x_{h}}^{1}} \\
& \leq C \sum_{j^{\prime} \geq j-4} \sum_{\substack{k^{\prime}, k^{\prime \prime} \geq k-2 \\
\left|k^{\prime}-k^{\prime \prime}\right| \leq 1}} 2^{k}\left\|S_{j^{\prime}+2}^{v} \dot{\Delta}_{k^{\prime}}^{h} a\right\|_{L_{x_{3}}^{\infty} L_{x_{h}}^{2}}\left\|\Delta_{j^{\prime}}^{v} \dot{\Delta}_{k^{\prime \prime}}^{h} b\right\|_{L^{2}} \\
& \leq C 2^{-j s} 2^{\left(1-\sigma_{1}-\sigma_{2}\right) k}\|a\|_{H^{\sigma_{1}, \frac{1}{2}-\alpha}}^{\frac{1}{2}}\|a\|_{H^{\sigma_{1}, \frac{1}{2}+\alpha}}^{\frac{1}{2}}\|b\|_{H^{\sigma_{2}, s}} \sum_{j^{\prime} \geq j-4} \sum_{k^{\prime} \geq k-2} 2^{-\left(\sigma_{1}+\sigma_{2}\right)\left(k^{\prime}-k\right)} 2^{-\left(j^{\prime}-j\right) s} c_{k^{\prime}} c_{j^{\prime}} \\
& \leq C c_{j, k} 2^{-j s} 2^{\left(1-\sigma_{1}-\sigma_{2}\right) k}\|a\|_{H^{\sigma_{1}, \frac{1}{2}-\alpha}}^{\frac{1}{2}}\|a\|_{H^{\sigma_{1}, \frac{1}{2}+\alpha}}^{\frac{1}{2}}\|b\|_{H^{\sigma_{2}, s}} .
\end{aligned}
$$

Summing up (4-5), (4-6), and (4-7), we obtain

$$
\left\|\Delta_{j}^{v} \dot{\Delta}_{k}^{h}\left(R_{a} b\right)\right\|_{L^{2}} \leq C c_{j, k} 2^{-j s} 2^{\left(1-\sigma_{1}-\sigma_{2}\right) k}\|a\|_{H^{\sigma_{1}, \frac{1}{2}-\alpha}}^{\frac{1}{2}}\|a\|_{H^{\sigma_{1}, \frac{1}{2}+\alpha}}^{\frac{1}{2}}\|b\|_{H^{\sigma_{2}, s}} .
$$

The lemma is proved in the case when the function $\Psi$ is identically 0 . In order to treat the general case, we only need to notice the fact that

$$
\left|\mathscr{F}\left[\Delta_{j} \dot{\Delta}_{k}^{h}\left(R_{a} b\right)\right]_{\Psi}(\xi)\right| \leq \mathscr{F}_{[}\left[\Delta_{j} \dot{\Delta}_{k}^{h}\left(R_{a_{\Psi}^{+}} b_{\Psi}^{+}\right)\right](\xi)
$$

As a consequence of Lemma 4.2 and (4-2), we have:

Lemma 4.3. Let $\frac{1}{2}>\alpha>0, s>0$, and $\sigma_{1}, \sigma_{2}<1$ such that $\sigma_{1}+\sigma_{2}>0$. Let $\left\{\tilde{\sigma}_{1}, \tilde{\sigma}_{2}\right\}=\left\{\sigma_{1}, \sigma_{2}\right\}$. Then

$$
\left\|(a b)_{\Psi}\right\|_{H^{\sigma_{1}+\sigma_{2}-1, s}} \leq C\left(\left\|a_{\Psi}\right\|_{H^{\sigma_{1}, \frac{1}{2}-\alpha}}^{\frac{1}{2}}\left\|a_{\Psi}\right\|_{H^{\sigma_{1}, \frac{1}{2}+\alpha}}^{\frac{1}{2}}\left\|b_{\Psi}\right\|_{H^{\sigma_{2}, s}}+\left\|a_{\Psi}\right\|_{H^{\tilde{\sigma}_{1}, s}}\left\|b_{\Psi}\right\|_{H^{\tilde{\sigma}_{2}, \frac{1}{2}-\alpha}}^{\frac{1}{2}}\left\|b_{\Psi}\right\|_{H^{\tilde{\sigma}_{2}, \frac{1}{2}+\alpha}}^{\frac{1}{2}}\right) .
$$

\section{Classical analytical-type estimates}

In this section, we prove Proposition 3.3. In this part, we don't need to use any regularizing effect from the analyticity, but only the fact that the $e^{\Phi\left(t, \xi_{3}\right)}$ is a sublinear function.

Notice that $\partial_{t} v_{\Phi}+a \lambda \dot{\theta}(t)\left\langle D_{3}\right\rangle v_{\Phi}=\left(\partial_{t} v\right)_{\Phi}$, we find from $\left(\mathrm{RNS}_{\varepsilon}\right)$ that

$$
\left\{\begin{array}{l}
\partial_{t} v_{\Phi}^{h}+a \lambda \dot{\theta}(t)\left\langle D_{3}\right\rangle v_{\Phi}^{h}-\Delta_{h} v_{\Phi}^{h}-\varepsilon^{2} \partial_{3}^{2} v_{\Phi}^{h}+\varepsilon^{\frac{1}{2}}\left(v \cdot \nabla v^{h}\right)_{\Phi}=-\nabla_{h} q_{\Phi}, \\
\partial_{t} v_{\Phi}^{3}+a \lambda \dot{\theta}(t)\left\langle D_{3}\right\rangle v_{\Phi}^{3}-\Delta_{h} v_{\Phi}^{3}-\varepsilon^{2} \partial_{3}^{2} v_{\Phi}^{3}+\varepsilon^{\frac{1}{2}}\left(v \cdot \nabla v^{3}\right)_{\Phi}=-\varepsilon^{2} \partial_{3} q_{\Phi} \\
\operatorname{div} v_{\Phi}=0 \\
v_{\Phi}(0)=e^{a\left\langle D_{3}\right\rangle} v_{0}(x)
\end{array}\right.
$$


Step 1. Estimates on the vertical component $\boldsymbol{v}_{\boldsymbol{\Phi}}^{\mathbf{3}}$. Noting that $\dot{\theta}(t) \geq 0$, we get from the second equation of (5-1) that

$$
\begin{aligned}
\frac{1}{2} \frac{d}{d t}\left\|v_{\Phi}^{3}(t)\right\|_{H^{-\frac{1}{2}, s}}^{2} & +\left\|\nabla_{h} v_{\Phi}^{3}(t)\right\|_{H^{-\frac{1}{2}, s}}^{2}+\left\|\varepsilon \partial_{3} v_{\Phi}^{3}(t)\right\|_{H^{-\frac{1}{2}, s}}^{2} \\
& \leq-\varepsilon^{\frac{1}{2}}\left(\left(v^{h} \cdot \nabla_{h} v^{3}\right)_{\Phi}, v_{\Phi}^{3}\right)_{H^{-\frac{1}{2}, s}}+\varepsilon^{\frac{1}{2}}\left(\left(v^{3} \operatorname{div}_{h} v^{h}\right)_{\Phi}, v_{\Phi}^{3}\right)_{H^{-\frac{1}{2}, s}}-\varepsilon^{2}\left(\partial_{3} q_{\Phi}, v_{\Phi}^{3}\right)_{H^{-\frac{1}{2}, s}} \\
& \stackrel{\text { def }}{=} \mathrm{I}+\mathrm{II}+\mathrm{III} .
\end{aligned}
$$

Here we used the fact that $\operatorname{div} v=0$, so $v \cdot \nabla v^{3}=v^{h} \cdot \nabla_{h} v^{3}-v^{3} \operatorname{div}_{h} v^{h}$.

For II, an application of Lemma 4.3 gives

$$
\begin{aligned}
|\mathrm{II}| & \leq \varepsilon^{\frac{1}{2}}\left\|\left(v^{3} \operatorname{div}_{h} v^{h}\right)_{\Phi}\right\|_{H^{-\frac{1}{2}, s}}\left\|v_{\Phi}^{3}\right\|_{H^{-\frac{1}{2}, s}} \\
& \leq C \varepsilon^{\frac{1}{2}}\left(\left\|v_{\Phi}^{3}\right\|_{H^{\frac{1}{2}, \frac{1}{2}-\alpha}}^{\frac{1}{2}}\left\|v_{\Phi}^{3}\right\|_{H^{\frac{1}{2}, \frac{1}{2}+\alpha}}^{\frac{1}{2}}\left\|\nabla_{h} v_{\Phi}^{h}\right\|_{H^{0, s}}+\left\|v_{\Phi}^{3}\right\|_{H^{\frac{1}{2}, s}}\left\|\nabla_{h} v_{\Phi}^{h}\right\|_{H^{0, \frac{1}{2}-\alpha}}^{\frac{1}{2}}\left\|\nabla_{h} v_{\Phi}^{h}\right\|_{H^{0, \frac{1}{2}+\alpha}}^{\frac{1}{2}}\right)\left\|v_{\Phi}^{3}\right\|_{H^{-\frac{1}{2}, s}} \\
& \leq C \dot{\theta}(t)\left\|v_{\Phi}^{3}\right\|_{H^{-\frac{1}{2}, s}}^{2}+\frac{1}{100} a\left\|\nabla_{h} v_{\Phi}^{h}\right\|_{H^{0, s}}^{2}+\frac{1}{100}\left\|v_{\Phi}^{3}\right\|_{H^{\frac{1}{2}, s}}^{2} .
\end{aligned}
$$

For I, we get by integration by parts that

$$
\mathrm{I}=\varepsilon^{\frac{1}{2}}\left(\left(\operatorname{div}_{h} v^{h} v^{3}\right)_{\Phi}, v_{\Phi}^{3}\right)_{H^{-\frac{1}{2}, s}}+\varepsilon^{\frac{1}{2}}\left(\left(v^{h} v^{3}\right)_{\Phi}, \nabla_{h} v_{\Phi}^{3}\right)_{H^{-\frac{1}{2}, s}} \stackrel{\text { def }}{=} \mathrm{I}_{1}+\mathrm{I}_{2} .
$$

As in (5-3), we have

$$
\left|\mathrm{I}_{1}\right| \leq C \dot{\theta}(t)\left\|v_{\Phi}^{3}\right\|_{H^{-\frac{1}{2}, s}}^{2}+\frac{1}{100} a\left\|\nabla_{h} v_{\Phi}^{h}\right\|_{H^{0, s}}^{2}+\frac{1}{100}\left\|v_{\Phi}^{3}\right\|_{H^{\frac{1}{2}, s}}^{2},
$$

and by Lemma 4.3,

$$
\begin{aligned}
\left|\mathrm{I}_{2}\right| & \leq \varepsilon^{\frac{1}{2}}\left\|\left(v^{3} v^{h}\right)_{\Phi}\right\|_{H^{-\frac{1}{2}, s}}\left\|\nabla_{h} v_{\Phi}^{3}\right\|_{H^{-\frac{1}{2}, s}} \\
& \leq C \varepsilon^{\frac{1}{2}}\left(\left\|v_{\Phi}^{h}\right\|_{H^{\frac{1}{2}, s}}\left\|v_{\Phi}^{3}\right\|_{H^{0, \frac{1}{2}-\alpha}}^{\frac{1}{2}}\left\|v_{\Phi}^{3}\right\|_{H^{0, \frac{1}{2}+\alpha}}^{\frac{1}{2}}+\left\|v_{\Phi}^{h}\right\|_{H^{0, \frac{1}{2}-\alpha}}^{\frac{1}{2}}\left\|v_{\Phi}^{h}\right\|_{H^{0, \frac{1}{2}+\alpha}}^{\frac{1}{2}}\left\|v_{\Phi}^{3}\right\|_{H^{\frac{1}{2}, s}}\right)\left\|\nabla_{h} v_{\Phi}^{3}\right\|_{H^{-\frac{1}{2}, s}} \\
& \leq C \Psi(t)\left(\varepsilon\left\|v_{\Phi}^{h}\right\|_{H^{\frac{1}{2}, s}}^{2}+\left\|v_{\Phi}^{3}\right\|_{H^{\frac{1}{2}, s}}^{2}\right)+\frac{1}{100}\left\|\nabla_{h} v_{\Phi}^{3}\right\|_{H^{-\frac{1}{2}, s}}^{2} .
\end{aligned}
$$

Now, we turn to the estimates of the pressure. Recall that

$$
-\Delta_{\varepsilon} p=\varepsilon^{\frac{1}{2}}\left(\partial_{i} \partial_{j}\left(v^{i} v^{j}\right)+\partial_{i} \partial_{3}\left(v^{i} v^{3}\right)-2 \partial_{3}\left(v^{3} \operatorname{div}_{h} v^{h}\right)\right) .
$$

Here and in what follows the indexes $i, j$ run from 1 to 2 . Thus, we can write $p=p^{1}+p^{2}+p^{3}$, with

$$
p^{1}=\varepsilon^{\frac{1}{2}}\left(-\Delta_{\varepsilon}\right)^{-1} \partial_{i} \partial_{j}\left(v^{i} v^{j}\right), \quad p^{2}=\varepsilon^{\frac{1}{2}}\left(-\Delta_{\varepsilon}\right)^{-1} \partial_{i} \partial_{3}\left(v^{i} v^{3}\right), \quad p^{3}=-2 \varepsilon^{\frac{1}{2}}\left(-\Delta_{\varepsilon}\right)^{-1} \partial_{3}\left(v^{3} \operatorname{div}_{h} v^{h}\right) .
$$

Integrating by parts, we get

$$
\varepsilon^{2}\left(\partial_{3} p_{\Phi}^{1}, v_{\Phi}^{3}\right)_{H^{-\frac{1}{2}, s}}=-\varepsilon\left(p_{\Phi}^{1}, \varepsilon \partial_{3} v_{\Phi}^{3}\right)_{H^{-\frac{1}{2}, s}} \leq C \varepsilon^{2}\left\|p_{\Phi}^{1}\right\|_{H^{-\frac{1}{2}, s}}^{2}+\frac{1}{100}\left\|\varepsilon \partial_{3} v_{\Phi}^{3}\right\|_{H^{-\frac{1}{2}, s}}^{2}
$$

which together with the fact that the operator $\partial_{i} \partial_{j}\left(-\Delta_{\varepsilon}\right)^{-1}$ is bounded on $H^{\sigma, s}$ together with Lemma 4.3 implies that

$$
\begin{aligned}
\varepsilon^{2}\left(\partial_{3} p_{\Phi}^{1}, v_{\Phi}^{3}\right)_{H^{-\frac{1}{2}, s}} & \leq C \varepsilon^{3}\left\|\left(v^{h} \otimes v^{h}\right)_{\Phi}\right\|_{H^{-\frac{1}{2}, s}}^{2}+\frac{1}{100}\left\|\varepsilon \partial_{3} v_{\Phi}^{3}\right\|_{H^{-\frac{1}{2}, s}}^{2} \\
& \leq C \varepsilon^{3}\left\|v_{\Phi}^{h}\right\|_{H^{\frac{1}{2}, s}}^{2}\left\|v_{\Phi}^{h}\right\|_{H^{0, \frac{1}{2}-\alpha}}\left\|v_{\Phi}^{h}\right\|_{H^{0, \frac{1}{2}+\alpha}}+\frac{1}{100}\left\|\varepsilon \partial_{3} v_{\Phi}^{3}\right\|_{H^{-\frac{1}{2}, s}}^{2} \\
& \leq C \varepsilon^{2} \Psi(t) \varepsilon\left\|v_{\Phi}^{h}\right\|_{H^{\frac{1}{2}, s}}^{2}+\frac{1}{100}\left\|\varepsilon \partial_{3} v_{\Phi}^{3}\right\|_{H^{-\frac{1}{2}, s}}^{2} .
\end{aligned}
$$


For the term containing $p_{2}$, we get by integration by parts that

$$
\varepsilon^{2}\left(\partial_{3} p_{\Phi}^{2}, v_{\Phi}^{3}\right)_{H^{-\frac{1}{2}, s}}=-\varepsilon^{\frac{1}{2}}\left(\varepsilon^{2} \partial_{3}^{2}\left(-\Delta_{\varepsilon}\right)^{-1}\left(v^{i} v^{3}\right)_{\Phi}, \partial_{i} v_{\Phi}^{3}\right)_{H^{-\frac{1}{2}, s}} .
$$

Using the fact that $\left(\varepsilon \partial_{3}\right)^{2}\left(-\Delta_{\varepsilon}\right)^{-1}$ is bounded on $H^{\sigma, s}$ together with Lemma 4.3 , we then have

$$
\begin{aligned}
\varepsilon^{2}\left(\partial_{3} p_{\Phi}^{2}, v_{\Phi}^{3}\right)_{H^{-\frac{1}{2}, s}} & \leq C \varepsilon^{\frac{1}{2}}\left\|\left(v^{3} v^{h}\right)_{\Phi}\right\|_{H^{-\frac{1}{2}, s}}\left\|\nabla_{h} v_{\Phi}^{3}\right\|_{H^{-\frac{1}{2}, s}} \\
& \leq C \Psi(t)\left(\varepsilon\left\|v_{\Phi}^{h}\right\|_{H^{\frac{1}{2}, s}}^{2}+\left\|v_{\Phi}^{3}\right\|_{H^{\frac{1}{2}, s}}^{2}\right)+\frac{1}{100}\left\|\nabla_{h} v_{\Phi}^{3}\right\|_{H^{-\frac{1}{2}, s}}^{2}
\end{aligned}
$$

For the last term, coming from $p_{3}$, we use again the fact that $\left(\varepsilon \partial_{3}\right)^{2}\left(-\Delta_{\varepsilon}\right)^{-1}$ is bounded on $H^{\sigma, s}$ and obtain

$$
\begin{aligned}
\varepsilon^{2}\left(\partial_{3} p_{\Phi}^{3}, v_{\Phi}^{3}\right)_{H^{-\frac{1}{2}, s}} & \leq C \varepsilon^{\frac{1}{2}}\left\|\left(v^{3} \operatorname{div} v^{h}\right)_{\Phi}\right\|_{H^{-\frac{1}{2}, s}}\left\|v_{\Phi}^{3}\right\|_{H^{-\frac{1}{2}, s}} \\
& \leq C \dot{\theta}(t)\left\|v_{\Phi}^{3}\right\|_{H^{-\frac{1}{2}, s}}^{2}+\frac{1}{100} a\left\|\nabla_{h} v_{\Phi}^{h}\right\|_{H^{0, s}}^{2}+\frac{1}{100}\left\|v_{\Phi}^{3}\right\|_{H^{\frac{1}{2}, s}}^{2} .
\end{aligned}
$$

Summing up (5-2)-(5-5) and (5-7)-(5-9), we obtain

$$
\begin{aligned}
& \frac{d}{d t}\left\|v_{\Phi}^{3}(t)\right\|_{H^{-\frac{1}{2}, s}}^{2}+\left\|v_{\Phi}^{3}(t)\right\|_{H^{\frac{1}{2}, s}}^{2} \\
& \quad \leq C \dot{\theta}(t)\left\|v_{\Phi}^{3}\right\|_{H^{-\frac{1}{2}, s}}^{2}+C \Psi(t)\left(\varepsilon\left\|v_{\Phi}^{h}\right\|_{H^{\frac{1}{2}, s}}^{2}+\left\|v_{\Phi}^{3}\right\|_{H^{\frac{1}{2}, s}}^{2}\right)+\frac{1}{20} a\left\|\nabla_{h} v_{\Phi}^{h}\right\|_{H^{0, s}}^{2},
\end{aligned}
$$

where we used the equality $\left\|\nabla_{h} v_{\Phi}^{3}\right\|_{H^{-\frac{1}{2}, s}}^{2}=\left\|v_{\Phi}^{3}\right\|_{H^{\frac{1}{2}, s}}^{2}$.

Step 2. Estimates on the horizontal component $\boldsymbol{v}_{\boldsymbol{\Phi}}^{\boldsymbol{h}}$. From the first equation of (5-1), we infer that

$$
\begin{aligned}
\frac{1}{2} \frac{d}{d t}\left\|\varepsilon^{\frac{1}{2}} v_{\Phi}^{h}(t)\right\|_{H^{-\frac{1}{2}, s}}^{2}+\left\|\varepsilon^{\frac{1}{2}} \nabla_{h} v_{\Phi}^{h}(t)\right\|_{H^{-\frac{1}{2}, s}}^{2} & +\varepsilon\left\|\varepsilon \partial_{3} v_{\Phi}^{h}(t)\right\|_{H^{-\frac{1}{2}, s}}^{2} \\
& \leq-\varepsilon\left(\left(v \cdot \nabla v^{h}\right)_{\Phi}, \varepsilon^{\frac{1}{2}} v_{\Phi}^{h}\right)_{H^{-\frac{1}{2}, s}}-\varepsilon\left(\nabla_{h} q_{\Phi}, v_{\Phi}^{h}\right)_{H^{-\frac{1}{2}, s}} \\
& \stackrel{\text { def }}{=} \mathrm{I}+\mathrm{II} .
\end{aligned}
$$

We rewrite I as

$$
\mathrm{I}=-\varepsilon\left(\left(v^{h} \cdot \nabla_{h} v^{h}\right)_{\Phi}, \varepsilon^{\frac{1}{2}} v_{\Phi}^{h}\right)_{H^{-\frac{1}{2}, s}}-\varepsilon\left(\left(v^{3} \partial_{3} v^{h}\right)_{\Phi}, \varepsilon^{\frac{1}{2}} v_{\Phi}^{h}\right)_{H^{-\frac{1}{2}, s}} \stackrel{\text { def }}{=} \mathrm{I}_{1}+\mathrm{I}_{2} .
$$

An application of Lemma 4.3 gives

$$
\begin{aligned}
\left|\mathrm{I}_{1}\right| & \leq \varepsilon\left\|\left(v^{h} \nabla_{h} v^{h}\right)_{\Phi}\right\|_{H^{-\frac{1}{2}, s}}\left\|\varepsilon^{\frac{1}{2}} v_{\Phi}^{h}\right\|_{H^{-\frac{1}{2}, s}} \\
& \leq C \varepsilon\left(\left\|v_{\Phi}^{h}\right\|_{H^{\frac{1}{2}, \frac{1}{2}-\alpha}}^{\frac{1}{2}}\left\|v_{\Phi}^{h}\right\|_{H^{\frac{1}{2}, \frac{1}{2}+\alpha}}^{\frac{1}{2}}\left\|\nabla_{h} v_{\Phi}^{h}\right\|_{H^{0, s}}+\left\|v_{\Phi}^{h}\right\|_{H^{\frac{1}{2}, s}}\left\|\nabla_{h} v_{\Phi}^{h}\right\|_{H^{0, \frac{1}{2}-\alpha}}^{\frac{1}{2}}\left\|\nabla_{h} v_{\Phi}^{h}\right\|_{H^{0, \frac{1}{2}+\alpha}}^{\frac{1}{2}}\left\|\varepsilon^{\frac{1}{2}} v_{\Phi}^{h}\right\|_{H^{-\frac{1}{2}, s}}\right. \\
& \leq C \dot{\theta}(t)\left\|\varepsilon^{\frac{1}{2}} v_{\Phi}^{h}\right\|_{H^{-\frac{1}{2}, s}}^{2}+\frac{1}{100} a\left\|\nabla_{h} v_{\Phi}^{h}\right\|_{H^{0, s}}^{2}+\frac{1}{100}\left\|\varepsilon^{\frac{1}{2}} v_{\Phi}^{h}\right\|_{H^{\frac{1}{2}, s}}^{2} .
\end{aligned}
$$

For $\mathrm{I}_{2}$, we use integration by parts and $\operatorname{div} v=0$ to get

$$
\mathrm{I}_{2}=-\varepsilon\left(\left(\operatorname{div}_{h} v^{h} v^{h}\right)_{\Phi}, \varepsilon^{\frac{1}{2}} v_{\Phi}^{h}\right)_{H^{-\frac{1}{2}, s}}+\left(\left(v^{h} v^{3}\right)_{\Phi}, \varepsilon^{\frac{1}{2}} \varepsilon \partial_{3} v_{\Phi}^{h}\right)_{H^{-\frac{1}{2}, s}} \stackrel{\text { def }}{=} \mathrm{I}_{21}+\mathrm{I}_{22}
$$

As in (5-12), we have

$$
\left|I_{21}\right| \leq C \dot{\theta}(t)\left\|\varepsilon^{\frac{1}{2}} v_{\Phi}^{h}\right\|_{H^{-\frac{1}{2}, s}}^{2}+\frac{1}{100} a\left\|\nabla_{h} v_{\Phi}^{h}\right\|_{H^{0, s}}^{2}+\frac{1}{100}\left\|\varepsilon^{\frac{1}{2}} v_{\Phi}^{h}\right\|_{H^{\frac{1}{2}, s}}^{2},
$$


and by Lemma 4.3,

$$
\begin{aligned}
\left|I_{22}\right| & \leq\left\|\left(v^{3} v^{h}\right)_{\Phi}\right\|_{H^{-\frac{1}{2}, s}} \varepsilon^{\frac{1}{2}}\left\|\varepsilon \partial_{3} v_{\Phi}^{h}\right\|_{H^{-\frac{1}{2}, s}} \\
& \leq C\left(\left\|v_{\Phi}^{h}\right\|_{H^{0, s}}\left\|v_{\Phi}^{3}\right\|_{H^{\frac{1}{2}, \frac{1}{2}-\alpha}}^{\frac{1}{2}}\left\|v_{\Phi}^{3}\right\|_{H^{\frac{1}{2}, \frac{1}{2}+\alpha}}^{\frac{1}{2}}+\left\|v_{\Phi}^{h}\right\|_{H^{0, \frac{1}{2}-\alpha}}^{\frac{1}{2}}\left\|v_{\Phi}^{h}\right\|_{H^{0, \frac{1}{2}+\alpha}}^{\frac{1}{2}}\left\|v_{\Phi}^{3}\right\|_{H^{\frac{1}{2}, s}}\right) \varepsilon^{\frac{1}{2}}\left\|\varepsilon \partial_{3} v_{\Phi}^{h}\right\|_{H^{-\frac{1}{2}, s}} \\
& \leq C a \dot{\theta}(t)\left\|v_{\Phi}^{h}\right\|_{H^{0, s}}^{2}+C \Psi(t)\left\|v_{\Phi}^{3}\right\|_{H^{\frac{1}{2}, s}}^{2}+\frac{\varepsilon}{100}\left\|\varepsilon \partial_{3} v_{\Phi}^{h}\right\|_{H^{-\frac{1}{2}, s} .}
\end{aligned}
$$

To deal with the pressure, we write $p=p^{1}+p^{2}+p^{3}$, with $p^{1}, p^{2}, p^{3}$ defined by (5-6). Using the boundedness of the operator $\partial_{i} \partial_{j}\left(-\Delta_{\varepsilon}\right)^{-1}$ on $H^{\sigma, s}$ together with Lemma 4.3, we have

$$
\begin{aligned}
\varepsilon\left(\nabla_{h} p_{\Phi}^{1}, v_{\Phi}^{h}\right)_{H^{-\frac{1}{2}, s}} & =-\varepsilon\left(\left(-\Delta_{\varepsilon}\right)^{-1} \partial_{i} \partial_{j}\left(v^{i} v^{j}\right)_{\Phi}, \varepsilon^{\frac{1}{2}} \operatorname{div}_{h} v_{\Phi}^{h}\right)_{H^{-\frac{1}{2}, s}} \\
& \leq C \varepsilon\left\|\left(v^{h} \otimes v^{h}\right)_{\Phi}\right\|_{H^{-\frac{1}{2}, s}}\left\|\varepsilon^{\frac{1}{2}} \nabla_{h} v_{\Phi}^{h}\right\|_{H^{-\frac{1}{2}, s}} \\
& \leq C \varepsilon^{\frac{1}{2}}\left\|v_{\Phi}^{h}\right\|_{H^{0, \frac{1}{2}-\alpha}}^{\frac{1}{2}}\left\|v_{\Phi}^{h}\right\|_{H^{0, \frac{1}{2}+\alpha}}^{\frac{1}{2}}\left\|\varepsilon^{\frac{1}{2}} v_{\Phi}^{h}\right\|_{H^{\frac{1}{2}, s}}\left\|\varepsilon^{\frac{1}{2}} \nabla_{h} v_{\Phi}^{h}\right\|_{H^{-\frac{1}{2}, s}} \\
& \leq C \Psi(t)\left\|\varepsilon^{\frac{1}{2}} v_{\Phi}^{h}\right\|_{H^{\frac{1}{2}, s}}^{2}+\frac{1}{100}\left\|\varepsilon^{\frac{1}{2}} \nabla_{h} v_{\Phi}^{h}\right\|_{H^{-\frac{1}{2}, s}}^{2} .
\end{aligned}
$$

For the term coming from $p_{2}$, we integrate by parts to get

$$
\varepsilon\left(\nabla_{h} p_{\Phi}^{2}, v_{\Phi}^{h}\right)_{H^{-\frac{1}{2}, s}}=-\left(\varepsilon \partial_{i} \partial_{3}\left(-\Delta_{\varepsilon}\right)^{-1}\left(v^{i} v^{3}\right)_{\Phi}, \varepsilon^{\frac{1}{2}} \operatorname{div}_{h} v_{\Phi}^{h}\right)_{H^{-\frac{1}{2}, s}},
$$

then note that $\varepsilon \partial_{3} \partial_{i}\left(-\Delta_{\varepsilon}\right)^{-1}$ is bounded on $H^{\sigma, s}$. We get, by Lemma 4.3,

$$
\begin{aligned}
\varepsilon\left(\nabla_{h} p_{\Phi}^{2}, v_{\Phi}^{h}\right)_{H^{-\frac{1}{2}, s}} & \leq C\left\|\left(v^{3} v^{h}\right)_{\Phi}\right\|_{H^{-\frac{1}{2}, s}}\left\|\varepsilon^{\frac{1}{2}} \nabla_{h} v_{\Phi}^{h}\right\|_{H^{-\frac{1}{2}, s}} \\
& \leq C a \dot{\theta}(t)\left\|v_{\Phi}^{h}\right\|_{H^{0, s}}^{2}+C \Psi(t)\left\|v_{\Phi}^{3}\right\|_{H^{\frac{1}{2}, s}}^{2}+\frac{1}{100}\left\|\varepsilon^{\frac{1}{2}} \nabla_{h} v_{\Phi}^{h}\right\|_{H^{-\frac{1}{2}, s}}^{2}
\end{aligned}
$$

Similarly,

$$
\begin{aligned}
\varepsilon\left(\nabla_{h} p_{\Phi}^{3},\right. & \left.v_{\Phi}^{h}\right)_{H^{-\frac{1}{2}, s}} \\
& \leq C\left\|\left(v^{3} \operatorname{div}{ }_{h} v^{h}\right)_{\Phi}\right\|_{H^{-\frac{1}{2}, s}}\left\|\varepsilon^{\frac{1}{2}} v_{\Phi}^{h}\right\|_{H^{-\frac{1}{2}, s}} \\
& \leq\left(\left\|v_{\Phi}^{3}\right\|_{H^{\frac{1}{2}, \frac{1}{2}-\alpha}}^{\frac{1}{2}}\left\|v_{\Phi}^{3}\right\|_{H^{\frac{1}{2}}, \frac{1}{2}+\alpha}^{\frac{1}{2}}\left\|\nabla_{h} v_{\Phi}^{h}\right\|_{H^{0, s}}+\left\|v_{\Phi}^{3}\right\| H^{\frac{1}{2}, s}\left\|\nabla_{h} v_{\Phi}^{h}\right\|_{H^{0, \frac{1}{2}-\alpha}}^{\frac{1}{2}}\left\|\nabla_{h} v_{\Phi}^{h}\right\|_{H^{0, \frac{1}{2}+\alpha}}^{\frac{1}{2}}\right)\left\|\varepsilon^{\frac{1}{2}} v_{\Phi}^{h}\right\|_{H^{-\frac{1}{2}, s}} \\
& \leq C \dot{\theta}(t)\left\|\varepsilon^{\frac{1}{2}} v_{\Phi}^{h}\right\|_{H^{-\frac{1}{2}, s}}^{2}+\frac{1}{100} a\left\|\nabla_{h} v_{\Phi}^{h}\right\|_{H^{0, s}}^{2}+\frac{1}{100}\left\|v_{\Phi}^{3}\right\|_{H^{\frac{1}{2}, s}}^{2}
\end{aligned}
$$

Summing up (5-11)-(5-17) yields

$$
\begin{aligned}
& \frac{d}{d t}\left\|\varepsilon^{\frac{1}{2}} v_{\Phi}^{h}(t)\right\|_{H^{-\frac{1}{2}, s}}^{2}+\left\|\varepsilon^{\frac{1}{2}} v_{\Phi}^{h}(t)\right\|_{H^{\frac{1}{2}, s}}^{2} \\
& \leq C \dot{\theta}(t)\left(\left\|\varepsilon^{\frac{1}{2}} v_{\Phi}^{h}\right\|_{H^{-\frac{1}{2}, s}}^{2}+a\left\|v_{\Phi}^{h}\right\|_{H^{0, s}}^{2}\right) \\
& \quad+C \Psi(t)\left(\left\|v_{\Phi}^{3}\right\|_{H^{\frac{1}{2}, s}}^{2}+\left\|\varepsilon^{\frac{1}{2}} v_{\Phi}^{h}\right\|_{H^{\frac{1}{2}, s}}^{2}\right)+\frac{1}{20} a\left\|\nabla_{h} v_{\Phi}^{h}\right\|_{H^{0, s}}^{2}+\frac{1}{20}\left\|v_{\Phi}^{3}\right\|_{H^{\frac{1}{2}, s}}^{2} .
\end{aligned}
$$

Now we are in a position to prove Proposition 3.3. Combining the energy estimate (5-10) with (5-18), we obtain

$$
\begin{aligned}
\frac{d}{d t}\left(\left\|\varepsilon^{\frac{1}{2}} v_{\Phi}^{h}(t)\right\|_{H^{-\frac{1}{2}, s}}^{2}+\|\right. & \left.v_{\Phi}^{3}(t) \|_{H^{-\frac{1}{2}, s}}^{2}\right)+\left(\left\|\varepsilon^{\frac{1}{2}} v_{\Phi}^{h}(t)\right\|_{H^{\frac{1}{2}, s}}^{2}+\left\|v_{\Phi}^{3}(t)\right\|_{H^{\frac{1}{2}, s}}^{2}\right) \\
\leq C \dot{\theta}(t)\left(\left\|\varepsilon^{\frac{1}{2}} v_{\Phi}^{h}\right\|_{H^{-\frac{1}{2}, s}}^{2}+\right. & \left.\left\|v_{\Phi}^{3}\right\|_{H^{-\frac{1}{2}, s}}^{2}\right)+C a \dot{\theta}(t)\left\|v_{\Phi}^{h}\right\|_{H^{0, s}}^{2} \\
& +C \Psi(t)\left(\left\|\varepsilon^{\frac{1}{2}} v_{\Phi}^{h}\right\|_{H^{-\frac{1}{2}, s}}^{2}+\left\|v_{\Phi}^{3}\right\|_{H^{\frac{1}{2}, s}}^{2}\right)+\frac{1}{10} a\left\|\nabla_{h} v_{\Phi}^{h}\right\|_{H^{0, s}}^{2}
\end{aligned}
$$


From this and Gronwall's inequality, it follows that

$$
\begin{aligned}
& \left\|\varepsilon^{\frac{1}{2}} v_{\Phi}^{h}(t)\right\|_{H^{-\frac{1}{2}, s}}^{2}+\left\|v_{\Phi}^{3}(t)\right\|_{H^{-\frac{1}{2}, s}}^{2}+\int_{0}^{t}\left(\left\|\varepsilon^{\frac{1}{2}} v_{\Phi}^{h}(\tau)\right\|_{H^{\frac{1}{2}, s}}^{2}+\left\|v_{\Phi}^{3}(\tau)\right\|_{H^{\frac{1}{2}, s}}^{2}\right) d \tau \\
& \leq \exp \left(C \int_{0}^{t} \dot{\theta}(\tau) d \tau\right)\left(\left\|e^{a\left\langle D_{3}\right\rangle} v_{0}\right\|_{H^{-\frac{1}{2}, s}}^{2}+a \int_{0}^{t} \dot{\theta}(\tau)\left\|v_{\Phi}^{h}(\tau)\right\|_{H^{0, s}}^{2} d+C \int_{0}^{t} \Psi(\tau)\left(\left\|\varepsilon^{\frac{1}{2}} v_{\Phi}^{h}(\tau)\right\|_{H^{\frac{1}{2}, s} \tau}^{2} \tau\right.\right. \\
& \left.\left.\quad+\left\|v_{\Phi}^{3}(\tau)\right\|_{H^{\frac{1}{2}, s}}^{2}\right) d \tau+\frac{1}{10} a \int_{0}^{t}\left\|\nabla_{h} v_{\Phi}^{h}(\tau)\right\|_{H^{0, s}}^{2} d \tau\right) .
\end{aligned}
$$

This finishes the proof of Proposition 3.3.

\section{Regularizing the effect of analyticity}

Let's now prove Proposition 3.4. Here we will encounter two kinds of bad terms, where we lose a vertical derivative. The first one is $\left(v^{3} \partial_{3} v^{h}\right)_{\Phi}$ and the second term comes from $-\nabla_{h} p$. In this last term, we really lose a vertical derivative. To compensate this loss, we use the divergence-free condition $\left(\partial_{3} u^{3}=-\operatorname{div}_{h} u^{h}\right)$ and more important, the fact that the equation contain an extra-regularizing term given by the analyticity of the solution.

Step 1. Estimates on the horizontal component $v_{\Phi}^{h}$. Let us recall that $v_{\Phi}^{h}$ verifies the equations

$$
\partial_{t} v_{\Phi}^{h}+a \lambda \dot{\theta}(t)\left\langle D_{3}\right\rangle v_{\Phi}^{h}-\Delta_{h} v_{\Phi}^{h}-\varepsilon^{2} \partial_{3}^{2} v_{\Phi}^{h}+\varepsilon^{\frac{1}{2}}\left(v \cdot \nabla v^{h}\right)_{\Phi}=-\nabla_{h} q_{\Phi}
$$

Note that $\dot{\theta} \geq 0$, we perform an energy estimate in $H^{0, s}$ to obtain

$$
\begin{aligned}
\frac{1}{2} \frac{d}{d t}\left\|v_{\Phi}^{h}\right\|_{H^{0, s}}^{2}+a \lambda \dot{\theta}(t)\left\|v_{\Phi}^{h}\right\|_{H^{0, s+\frac{1}{2}}}^{2} & +\left\|\nabla_{h} v_{\Phi}^{h}\right\|_{H^{0, s}}^{2}+\left\|\varepsilon \partial_{3} v_{\Phi}^{h}\right\|_{H^{0, s}}^{2} \\
& \leq \varepsilon^{\frac{1}{2}}\left(\left(v^{h} \otimes v^{h}\right)_{\Phi}, \nabla_{h} v_{\Phi}^{h}\right)_{H^{0, s}}-\varepsilon^{\frac{1}{2}}\left(\partial_{3}\left(v^{3} v^{h}\right)_{\Phi}, v_{\Phi}^{h}\right)_{H^{0, s}}-\left(\nabla_{h} p_{\Phi}, v_{\Phi}^{h}\right)_{H^{0, s}} \\
& \stackrel{\text { def }}{=} \mathrm{I}+\mathrm{II}+\mathrm{III} .
\end{aligned}
$$

We get by Lemma 4.3 and interpolation that

$$
\begin{aligned}
& |\mathrm{I}| \leq C \varepsilon^{\frac{1}{2}}\left\|\left(v^{h} \otimes v^{h}\right)_{\Phi}\right\|_{H^{0, s}}\left\|\nabla_{h} v_{\Phi}^{h}\right\|_{H^{0, s}} \\
& \leq C \varepsilon^{\frac{1}{2}}\left\|v_{\Phi}^{h}\right\|_{H \frac{1}{2}, \frac{1}{2}-\alpha}^{\frac{1}{2}}\left\|v_{\Phi}^{h}\right\|_{H^{\frac{1}{2}}, \frac{1}{2}+\alpha}^{\frac{1}{2}}\left\|v_{\Phi}^{h}\right\|_{H \frac{1}{2}, s}\left\|\nabla_{h} v_{\Phi}^{h}\right\|_{H^{0, s}} \\
& \leq C \varepsilon\left\|v_{\Phi}^{h}\right\|_{H \frac{1}{2}, \frac{1}{2}-\alpha}\left\|v_{\Phi}^{h}\right\|_{H \frac{1}{2}, \frac{1}{2}+\alpha}\left\|v_{\Phi}^{h}\right\|_{H \frac{1}{2}, s}^{2}+\frac{1}{100}\left\|\nabla_{h} v_{\Phi}^{h}\right\|_{H^{0, s}} \\
& \leq C \varepsilon\left\|v_{\Phi}^{h}\right\|_{H^{0, \frac{1}{2}-\alpha}}^{\frac{1}{2}}\left\|v_{\Phi}^{h}\right\|_{H^{0, \frac{1}{2}+\alpha}}^{\frac{1}{2}}\left\|\nabla_{h} v_{\Phi}^{h}\right\|_{H^{0, \frac{1}{2}-\alpha}}^{\frac{1}{2}}\left\|\nabla_{h} v_{\Phi}^{h}\right\|_{H^{0, \frac{1}{2}+\alpha}}^{\frac{1}{2}}\left\|v_{\Phi}^{h}\right\|_{H^{0, s}}\left\|v_{\Phi}^{h}\right\|_{H^{1, s}}+\frac{1}{100}\left\|\nabla_{h} v_{\Phi}^{h}\right\|_{H^{0, s}}^{2} \\
& \leq C \Psi(t)\left\|v_{\Phi}^{h}\right\|_{H^{1, s}}^{2}+C \dot{\theta}(t)\left\|v_{\Phi}^{h}\right\|_{H^{0, s}}^{2}+\frac{1}{100}\left\|\nabla_{h} v_{\Phi}^{h}\right\|_{H^{0, s}}^{2} \text {. }
\end{aligned}
$$

To estimate II, we use Bony's decomposition (4-3) to rewrite it as

$$
\mathrm{II}=-\varepsilon^{\frac{1}{2}}\left(\partial_{3}\left(T_{v^{h}}^{v} v_{3}\right)_{\Phi}, v_{\Phi}^{h}\right)_{H^{0, s}}-\varepsilon^{\frac{1}{2}}\left(\partial_{3}\left(R_{v_{3}}^{v} v^{h}\right)_{\Phi}, v_{\Phi}^{h}\right)_{H^{0, s}} \stackrel{\text { def }}{=} \mathrm{II}_{1}+\mathrm{II}_{2}
$$


From the proof of Lemma 4.2, it is easy to find that

$$
\begin{aligned}
\left|\mathrm{II}_{2}\right| & \leq C\left\|\left|D_{3}\right|^{\frac{1}{2}}\left(R_{v^{3}}^{v} v^{h}\right)_{\Phi}\right\|_{H^{-\frac{1}{2}, s}}\left\|\left|\varepsilon D_{3}\right|^{\frac{1}{2}}\left|D_{h}\right|^{\frac{1}{2}} v_{\Phi}^{h}\right\|_{H^{0, s}} \\
& \leq C\left\|v_{\Phi}^{3}\right\|_{H}^{\frac{1}{2}, \frac{1}{2}-\alpha}\left\|v_{\Phi}^{3}\right\|_{H \frac{1}{2}, \frac{1}{2}+\alpha}^{\frac{1}{2}}\left\|v_{\Phi}^{h}\right\|_{H^{0, s+\frac{1}{2}}}\left\|\nabla_{\varepsilon} v^{h}\right\|_{H^{0, s}} \\
& \leq C a \dot{\theta}(t)\left\|v_{\Phi}^{h}\right\|_{H^{0, s+\frac{1}{2}}}^{2}+\frac{1}{100}\left\|\nabla_{\varepsilon} v^{h}\right\|_{H^{0, s}}^{2} .
\end{aligned}
$$

Due to $\operatorname{div} v=0$, we rewrite $\mathrm{II}_{1}$ as

$$
\mathrm{II}_{1}=\varepsilon^{\frac{1}{2}}\left(\left(T_{v^{h}}^{v} \operatorname{div}_{h} v^{h}\right)_{\Phi}, v_{\Phi}^{h}\right)_{H^{0, s}}-\varepsilon^{\frac{1}{2}}\left(\left(T_{\partial_{3} v^{h}}^{v} v^{3}\right)_{\Phi}, v_{\Phi}^{h}\right)_{H^{0, s}} \stackrel{\text { def }}{=} \mathrm{II}_{11}+\mathrm{II}_{12} .
$$

Using Lemma 4.2 and interpolation, we have

$$
\begin{aligned}
\left|\mathrm{II}_{11}\right| & \leq \varepsilon^{\frac{1}{2}}\left\|\left(T_{v^{h}}^{v} \operatorname{div}_{h} v^{h}\right)_{\Phi}\right\|_{H^{-\frac{1}{2}, s}}\left\|v_{\Phi}^{h}\right\|_{H^{\frac{1}{2}, s}} \\
& \leq C \varepsilon^{\frac{1}{2}}\left\|v_{\Phi}^{h}\right\|_{H^{\frac{1}{2}, \frac{1}{2}-\alpha}}^{\frac{1}{2}}\left\|v_{\Phi}^{h}\right\|_{H^{\frac{1}{2}, \frac{1}{2}+\alpha}}^{\frac{1}{2}}\left\|\nabla_{h} v_{\Phi}^{h}\right\|_{H^{0, s}}\left\|v_{\Phi}^{h}\right\|_{H^{\frac{1}{2}, s}} \\
& \leq C \Psi(t)\left\|v_{\Phi}^{h}\right\|_{H^{1, s}}^{2}+C \dot{\theta}(t)\left\|v_{\Phi}^{h}\right\|_{H^{0, s}}^{2}+\frac{1}{100}\left\|\nabla_{h} v_{\Phi}^{h}\right\|_{H^{0, s}}^{2} .
\end{aligned}
$$

From the proof of Lemma 4.2, and using the fact that $s<1$, we can conclude that

$$
\begin{aligned}
\left|\mathrm{II}_{12}\right| & \leq C\left\|v_{\Phi}^{h}\right\|_{H^{0, s+\frac{1}{2}}}\left\|v_{\Phi}^{3}\right\|_{H^{\frac{1}{2}, s}}\left\|\nabla_{\varepsilon} v^{h}\right\|_{H^{0, s}} \\
& \leq C\left\|v_{\Phi}^{h}\right\|_{H^{0, s+\frac{1}{2}}}^{\frac{1}{2}}\left\|v_{\Phi}^{3}\right\|_{H^{\frac{1}{2}, \frac{1}{2}-\alpha}}^{\frac{1}{2}}\left\|v_{\Phi}^{3}\right\|_{H^{\frac{1}{2}, \frac{1}{2}+\alpha}}^{\frac{1}{2}}\left\|\nabla_{\varepsilon} v^{h}\right\|_{H^{0, s}} \\
& \leq C a \dot{\theta}(t)\left\|v_{\Phi}^{h}\right\|_{H^{0, s+\frac{1}{2}}}^{2}+\frac{1}{100}\left\|\nabla_{\varepsilon} v^{h}\right\|_{H^{0, s}}^{2} .
\end{aligned}
$$

We next turn to the estimate of the pressure. Recall that $p=p^{1}+p^{2}+p^{3}$ with $p^{1}, p^{2}, p^{3}$ defined by (5-6). Using the boundedness of $\left(-\Delta_{\varepsilon}\right)^{-1} \partial_{i} \partial_{j}$ on $H^{\sigma, s}$ together with Lemma 4.3, we get

$$
\begin{aligned}
\left(\nabla_{h} p_{\Phi}^{1}, v_{\Phi}^{h}\right)_{H^{0, s}} & =-\varepsilon^{\frac{1}{2}}\left(\left(-\Delta_{\varepsilon}\right)^{-1} \partial_{i} \partial_{j}\left(v^{i} v^{j}\right)_{\Phi}, \operatorname{div} v_{\Phi}^{h}\right)_{H^{0, s}} \\
& \leq C \varepsilon^{\frac{1}{2}}\left\|\left(v^{h} \otimes v^{h}\right)_{\Phi}\right\|_{H^{0, s}}\left\|\nabla_{h} v_{\Phi}^{h}\right\|_{H^{0, s}} \\
& \leq C \Psi(t)\left\|v_{\Phi}^{h}\right\|_{H^{1, s}}^{2}+C \dot{\theta}(t)\left\|v_{\Phi}^{h}\right\|_{H^{0, s}}^{2}+\frac{1}{100}\left\|\nabla_{h} v_{\Phi}^{h}\right\|_{H^{0, s}}^{2} .
\end{aligned}
$$

Notice that $\partial_{i} \partial_{j}\left(-\Delta_{\varepsilon}\right)^{-1}$ is bounded on $H^{\sigma, s}$. Exactly as in the estimate of II, we obtain

$$
\left(\nabla_{h} p_{\Phi}^{2}, v_{\Phi}^{h}\right)_{H^{0, s}} \leq C \Psi(t)\left\|v_{\Phi}^{h}\right\|_{H^{1, s}}^{2}+C \dot{\theta}(t)\left\|v_{\Phi}^{h}\right\|_{H^{0, s}}^{2}+C a \dot{\theta}(t)\left\|v_{\Phi}^{h}\right\|_{H^{0, s+\frac{1}{2}}}^{2}+\frac{1}{100}\left\|\nabla_{\varepsilon} v^{h}\right\|_{H^{0, s}}^{2} .
$$

We write

$$
\nabla_{h} p_{3}=-2 \partial_{3}\left|D_{3}\right|^{-\frac{1}{2}}\left(\nabla_{h}\left|D_{h}\right|^{\frac{1}{2}}\left|\varepsilon D_{3}\right|^{1 / 2}\left(-\Delta_{\varepsilon}\right)^{-1}\right)\left|D_{h}\right|^{-\frac{1}{2}}\left(v^{3} \operatorname{div}_{h} v^{h}\right)
$$

thus,

$$
\left(\nabla_{h} p_{\Phi}^{3}, v_{\Phi}^{h}\right)_{H^{0, s}}=-2\left(\left(\nabla_{h}\left|D_{h}\right|^{\frac{1}{2}}\left|\varepsilon D_{3}\right|^{\frac{1}{2}}\left(-\Delta_{\varepsilon}\right)^{-1}\right)\left|D_{h}\right|^{-\frac{1}{2}}\left(v^{3} \operatorname{div}_{h} v^{h}\right), \partial_{3}\left\langle D_{3}\right\rangle^{-\frac{1}{2}} v_{\Phi}^{h}\right)_{H^{0, s}} .
$$

Note that $\nabla_{h}\left|D_{h}\right|^{\frac{1}{2}}\left|\varepsilon D_{3}\right|^{\frac{1}{2}}\left(-\Delta_{\varepsilon}\right)^{-1}$ is a bounded operator on $H^{\sigma, s}$. Thus we get, by Lemma 4.3, $\left(\nabla_{h} p_{\Phi}^{3}, v_{\Phi}^{h}\right)_{H^{0, s}}$

$$
\begin{aligned}
& \leq C\left\|\left|D_{h}\right|^{-\frac{1}{2}}\left(v^{3} \operatorname{div}_{h} v^{h}\right)\right\|_{H^{0, s}}\left\|\partial_{3}\left\langle D_{3}\right\rangle^{-\frac{1}{2}} v_{\Phi}^{h}\right\|_{H^{0, s}} \\
& \leq C\left(\left\|v_{\Phi}^{3}\right\|_{H}^{\frac{1}{2}, \frac{1}{2}-\alpha}\left\|v_{\Phi}^{3}\right\|_{H \frac{1}{2}, \frac{1}{2}+\alpha}^{\frac{1}{2}}\left\|\nabla_{h} v_{\Phi}^{h}\right\|_{H^{0, s}}+\left\|v_{\Phi}^{3}\right\|_{H^{\frac{1}{2}, s}}\left\|\nabla_{h} v_{\Phi}^{h}\right\|_{H^{0, \frac{1}{2}-\alpha}}^{\frac{1}{2}}\left\|\nabla_{h} v_{\Phi}^{h}\right\|_{H^{0, \frac{1}{2}+\alpha}}^{\frac{1}{2}}\right)\left\|v^{h}\right\|_{H^{0, s+\frac{1}{2}}} \\
& \leq C a \dot{\theta}(t)\left\|v_{\Phi}^{h}\right\|_{H^{0, s+\frac{1}{2}}}^{2}+\frac{1}{100}\left\|\nabla_{h} v_{\Phi}^{h}\right\|_{H^{0, s}}^{2}+\frac{1}{100} a^{-1}\left\|v_{\Phi}^{3}\right\|_{H^{\frac{1}{2}, s}}^{2} .
\end{aligned}
$$


Summing up (6-1)-(6-8), we get by taking $\lambda$ big enough that

$$
\frac{d}{d t}\left\|v_{\Phi}^{h}(t)\right\|_{H^{0, s}}^{2}+\left\|\nabla_{h} v_{\Phi}^{h}(t)\right\|_{H^{0, s}}^{2} \leq C \dot{\theta}(t)\left\|v_{\Phi}^{h}\right\|_{H^{0, s}}^{2}+C \Psi(t)\left\|v_{\Phi}^{h}\right\|_{H^{1, s}}^{2}+\frac{1}{20} a^{-1}\left\|v_{\Phi}^{3}\right\|_{H^{\frac{1}{2}, s}}^{2} .
$$

Step 2. Estimates on the vertical component $\boldsymbol{v}_{\boldsymbol{\Phi}}^{\mathbf{3}}$. Recall that $v_{\Phi}^{3}$ satisfies

$$
\partial_{t} v_{\Phi}^{3}+\lambda a \dot{\theta}(t)\left\langle D_{3}\right\rangle v_{\Phi}^{3}-\Delta_{h} v_{\Phi}^{3}-\varepsilon^{2} \partial_{3}^{2} v_{\Phi}^{3}+\varepsilon^{\frac{1}{2}}\left(v \cdot \nabla v^{3}\right)_{\Phi}=-\varepsilon^{2} \partial_{3} q_{\Phi} .
$$

We perform an energy estimate in $H^{0, s}$ to obtain

$$
\begin{aligned}
\frac{1}{2} \frac{d}{d t}\left\|v_{\Phi}^{3}\right\|_{H^{0, s}}^{2}+\left\|\nabla_{h} v_{\Phi}^{3}\right\|_{H^{0, s}}^{2} & +\left\|\varepsilon \partial_{3} v_{\Phi}^{3}\right\|_{H^{0, s}}^{2} \\
& \leq-\varepsilon^{\frac{1}{2}}\left(\left(v^{h} \cdot \nabla_{h} v^{3}\right)_{\Phi}, v_{\Phi}^{3}\right)_{H^{0, s}}+\varepsilon^{\frac{1}{2}}\left(\left(v^{3} \operatorname{div}_{h} v^{h}\right)_{\Phi}, v_{\Phi}^{3}\right)_{H^{0, s}}-\varepsilon^{2}\left(\partial_{3} p_{\Phi}, v_{\Phi}^{3}\right)_{H^{0, s}} \\
& \stackrel{\operatorname{def}}{=} \mathrm{I}+\mathrm{II}+\mathrm{III} .
\end{aligned}
$$

Using Lemma 4.3 and interpolation, we have

$$
\begin{aligned}
& |\mathrm{I}| \leq C \varepsilon^{\frac{1}{2}}\left\|\left(v^{h} \cdot \nabla_{h} v^{3}\right)_{\Phi}\right\|_{H^{-\frac{1}{2}, s}}\left\|v_{\Phi}^{3}\right\|_{H^{\frac{1}{2}}, s} \\
& \leq C \varepsilon^{\frac{1}{2}}\left(\left\|v_{\Phi}^{h}\right\|_{H^{\frac{1}{2}, \frac{1}{2}-\alpha}}^{\frac{1}{2}}\left\|v_{\Phi}^{h}\right\|_{H^{\frac{1}{2}}, \frac{1}{2}+\alpha}^{\frac{1}{2}}\left\|\nabla_{h} v_{\Phi}^{3}\right\|_{H^{0, s}}+\left\|v_{\Phi}^{h}\right\|_{H \frac{1}{2}, s}\left\|\nabla_{h} v_{\Phi}^{3}\right\|_{H^{0, \frac{1}{2}-\alpha}}^{\frac{1}{2}}\left\|\nabla_{h} v_{\Phi}^{3}\right\|_{H^{0, \frac{1}{2}+\alpha}}^{\frac{1}{2}}\right)\left\|v_{\Phi}^{3}\right\|_{H^{\frac{1}{2}, s}} \\
& \leq C \Psi(t)\left\|v_{\Phi}\right\|_{H^{1, s}}^{2}+C \dot{\theta}(t)\left\|v_{\Phi}\right\|_{H^{0, s}}^{2}+\frac{1}{100}\left\|\nabla_{h} v_{\Phi}\right\|_{H^{0, s}}^{2} \text {, }
\end{aligned}
$$

and similarly,

$$
\begin{aligned}
|\mathrm{II}| & \leq C \varepsilon^{\frac{1}{2}}\left\|\left(v^{3} \operatorname{div}_{h} v^{h}\right)_{\Phi}\right\|_{H^{-\frac{1}{2}, s}}\left\|v_{\Phi}^{3}\right\|_{H^{\frac{1}{2}, s}} \\
& \leq C \Psi(t)\left\|v_{\Phi}^{3}\right\|_{H^{1, s}}^{2}+C \dot{\theta}(t)\left\|v_{\Phi}^{3}\right\|_{H^{0, s}}^{2}+\frac{1}{100}\left\|\nabla_{h} v_{\Phi}\right\|_{H^{0, s}}^{2} .
\end{aligned}
$$

Using the decomposition (5-6), we can similarly obtain

$$
|\mathrm{III}| \leq C \Psi(t)\left\|v_{\Phi}\right\|_{H^{1, s}}^{2}+C \dot{\theta}(t)\left\|v_{\Phi}\right\|_{H^{0, s}}^{2}+\frac{1}{100}\left\|\nabla_{h} v_{\Phi}\right\|_{H^{0, s}}^{2} .
$$

Summing up (6-10)-(6-13), we obtain

$$
\frac{d}{d t}\left\|v_{\Phi}^{3}\right\|_{H^{0, s}}^{2}+\left\|\nabla_{h} v_{\Phi}^{3}\right\|_{H^{0, s}}^{2} \leq C \Psi(t)\left\|v_{\Phi}\right\|_{H^{1, s}}^{2}+C \dot{\theta}(t)\left\|v_{\Phi}\right\|_{H^{0, s}}^{2}+\frac{1}{20}\left\|\nabla_{h} v_{\Phi}^{h}\right\|_{H^{0, s}}^{2} .
$$

Now we combine (6-9) with (6-14) to obtain

$$
\frac{d}{d t}\left\|v_{\Phi}\right\|_{H^{0, s}}^{2}+\left\|\nabla_{h} v_{\Phi}\right\|_{H^{0, s}}^{2} \leq C \dot{\theta}(t)\left\|v_{\Phi}\right\|_{H^{0, s}}^{2}+C \Psi(t)\left\|v_{\Phi}\right\|_{H^{1, s}}^{2}+\frac{1}{10 a}\left\|v_{\Phi}^{3}\right\|_{H^{\frac{1}{2}, s}}^{2} .
$$

From this and Gronwall's inequality, we infer that

$$
\begin{aligned}
\left\|v_{\Phi}(t)\right\|_{H^{0, s}}^{2} & +\int_{0}^{t}\left\|\nabla_{h} v_{\Phi}(\tau)\right\|_{H^{0, s}}^{2} d \tau \\
& \leq \exp \left(C \int_{0}^{t} \dot{\theta}(\tau) d \tau\right)\left(\left\|e^{a\left\langle D_{3}\right\rangle} v_{0}\right\|_{H^{0, s}}^{2}+\int_{0}^{t} \Psi(\tau)\left\|v_{\Phi}(\tau)\right\|_{H^{1, s}}^{2} d \tau+\frac{1}{10 a} \int_{0}^{t}\left\|v_{\Phi}^{3}(\tau)\right\|_{H^{\frac{1}{2}}, s}^{2} d \tau\right)
\end{aligned}
$$

This finishes the proof of Proposition 3.4. 


\section{Acknowledgments}

This work was partly done when Zhifei Zhang was visiting the Department of Mathematics of ParisSud University as a postdoctor fellow. Zhang would like to thank the department for its hospitality and support. The authors are indebted to the referee for fruitful remarks.

\section{References}

[Bony 1981] J.-M. Bony, "Calcul symbolique et propagation des singularités pour les équations aux dérivées partielles non linéaires", Ann. Sci. École Norm. Sup. (4) 14:2 (1981), 209-246. MR 84h:35177 Zbl 0495.35024

[Cannone et al. 1994] M. Cannone, Y. Meyer, and F. Planchon, "Solutions auto-similaires des équations de Navier-Stokes", exposé VIII in Séminaire sur les Équations aux Dérivées Partielles, 1993-1994, École Polytech., Palaiseau, 1994. MR 95k:35157

[Chemin 2004] J.-Y. Chemin, "Le système de Navier-Stokes incompressible soixante dix ans après Jean Leray", pp. 99-123 in Actes des Journées Mathématiques à la Mémoire de Jean Leray, edited by L. Guillopé and R. Didier, Sémin. Congr. 9, Soc. Math. France, Paris, 2004. MR 2006m:35274 Zbl 1075.35035

[Chemin and Gallagher 2006] J.-Y. Chemin and I. Gallagher, "On the global wellposedness of the 3-D Navier-Stokes equations with large initial data", Ann. Sci. École Norm. Sup. (4) 39:4 (2006), 679-698. MR 2008h:35265 Zbl 1124.35052

[Chemin and Gallagher 2009] J.-Y. Chemin and I. Gallagher, "Wellposedness and stability results for the Navier-Stokes equations in $\mathbf{R}^{3}$ ", Ann. Inst. H. Poincaré Anal. Non Linéaire 26:2 (2009), 599-624. MR 2010i:35278 Zbl 1165.35038

[Chemin and Gallagher 2010] J.-Y. Chemin and I. Gallagher, "Large, global solutions to the Navier-Stokes equations, slowly varying in one direction", Trans. Amer. Math. Soc. 362:6 (2010), 2859-2873. MR 2592939 Zbl 1189.35220

[Chemin and Zhang 2007] J.-Y. Chemin and P. Zhang, "On the global wellposedness to the 3-D incompressible anisotropic Navier-Stokes equations", Comm. Math. Phys. 272:2 (2007), 529-566. MR 2008j:35137 Zbl 1132.35068

[Chemin et al. 2000] J.-Y. Chemin, B. Desjardins, I. Gallagher, and E. Grenier, "Fluids with anisotropic viscosity", M2AN Math. Model. Numer. Anal. 34:2 (2000), 315-335. MR 2001m:35261 Zbl 0954.76012

[Chemin et al. 2006] J.-Y. Chemin, B. Desjardins, I. Gallagher, and E. Grenier, Mathematical geophysics. An introduction to rotating fluids and the Navier-Stokes equations, Oxford Lecture Series in Mathematics and its Applications 32, The Clarendon Press Oxford University Press, Oxford, 2006. MR 2007m:76148 Zbl 05029231

[Chemin et al. 2011] J.-Y. Chemin, I. Gallagher, and M. Paicu, "Global regularity for some classes of large solutions to the Navier-Stokes equations", Ann. Math. 173:2 (2011).

[Fujita and Kato 1964] H. Fujita and T. Kato, "On the Navier-Stokes initial value problem. I", Arch. Rational Mech. Anal. 16 (1964), 269-315. MR 29\#3774 Zbl 0126.42301

[Gallagher et al. 2003] I. Gallagher, D. Iftimie, and F. Planchon, "Asymptotics and stability for global solutions to the NavierStokes equations", Ann. Inst. Fourier (Grenoble) 53:5 (2003), 1387-1424. MR 2005b:35219 Zbl 1038.35054

[Kato 1984] T. Kato, "Strong $L^{p}$-solutions of the Navier-Stokes equation in $\mathbf{R}^{m}$, with applications to weak solutions", Math. Z. 187:4 (1984), 471-480. MR 86b:35171 Zbl 0545.35073

[Koch and Tataru 2001] H. Koch and D. Tataru, "Well-posedness for the Navier-Stokes equations", Adv. Math. 157:1 (2001), 22-35. MR 2001m:35257 Zbl 0972.35084

[Leray 1934] J. Leray, “Sur le mouvement d'un liquide visqueux emplissant l'espace”, Acta Math. 63 (1934), 193-248.

[Makhalov and Nikolaenko 2003] A. S. Makhalov and V. P. Nikolaenko, "Global solvability of three-dimensional NavierStokes equations with uniformly high initial vorticity", Uspekhi Mat. Nauk 58:2(350) (2003), 79-110. In Russian: translated in Russian Math. Surveys, 58 2003, 287-318. MR 2004h:35177 Zbl 1059.35099

[Pedlovsky 1979] J. Pedlovsky, Geophysical fluid dynamics, Springer, 1979.

[Raugel and Sell 1993] G. Raugel and G. R. Sell, "Navier-Stokes equations on thin 3D domains, I: Global attractors and global regularity of solutions", J. Amer. Math. Soc. 6:3 (1993), 503-568. MR 93j:35134

[Sammartino and Caflisch 1998] M. Sammartino and R. E. Caflisch, "Zero viscosity limit for analytic solutions, of the NavierStokes equation on a half-space. I. Existence for Euler and Prandtl equations”, Comm. Math. Phys. 192:2 (1998), 433-461. MR 99d:35129a 
[Ukhovskii and Iudovich 1968] M. R. Ukhovskii and V. I. Iudovich, "Axially symmetric flows of ideal and viscous fluids filling the whole space", J. Appl. Math. Mech. 32 (1968), 52-61. MR 39 \#650

[Weissler 1980] F. B. Weissler, "The Navier-Stokes initial value problem in $L^{p}$ ", Arch. Rational Mech. Anal. 74:3 (1980), 219-230. MR 83k:35071 Zbl 0454.35072

Received 27 Mar 2009. Revised 14 May 2010. Accepted 1 Sep 2010.

MARIUS PAICU: Marius.Paicu@math.u-psud.fr

Departement de Mathématique, Université Paris Sud, 91405 Orsay Cedex, France

http://www.math.u-psud.fr/ paicu/

ZHIFEI ZHANG: zfzhang@math.pku.edu.cn

School of Mathematical Sciences, Peking University, 100871 Beijing, China 


\section{Analysis \& PDE}

pjm.math.berkeley.edu/apde

EDITORS

EDITOR-IN-CHIEF

Maciej Zworski

University of California

Berkeley, USA

BOARD OF EDITORS

\begin{tabular}{|c|c|c|c|}
\hline Michael Aizenman & $\begin{array}{l}\text { Princeton University, USA } \\
\text { aizenman@math.princeton.edu }\end{array}$ & Nicolas Burq & $\begin{array}{l}\text { Université Paris-Sud 11, France } \\
\text { nicolas.burq@math.u-psud.fr }\end{array}$ \\
\hline Luis A. Caffarelli & $\begin{array}{l}\text { University of Texas, USA } \\
\text { caffarel@ math.utexas.edu }\end{array}$ & un-Yung Alice Chang & $\begin{array}{l}\text { Princeton University, USA } \\
\text { chang@math.princeton.edu }\end{array}$ \\
\hline Michael Christ & $\begin{array}{l}\text { University of California, Berkeley, USA } \\
\text { mchrist@math.berkeley.edu }\end{array}$ & Charles Fefferman & $\begin{array}{l}\text { Princeton University, USA } \\
\text { cf@math.princeton.edu }\end{array}$ \\
\hline Ursula Hamenstaedt & $\begin{array}{l}\text { Universität Bonn, Germany } \\
\text { ursula@math.uni-bonn.de }\end{array}$ & Nigel Higson & $\begin{array}{l}\text { Pennsylvania State Univesity, USA } \\
\text { higson@math.psu.edu }\end{array}$ \\
\hline Vaughan Jones & $\begin{array}{l}\text { University of California, Berkeley, USA } \\
\text { vfr@math.berkeley.edu }\end{array}$ & Herbert Koch & $\begin{array}{l}\text { Universität Bonn, Germany } \\
\text { koch@math.uni-bonn.de }\end{array}$ \\
\hline Izabella Laba & $\begin{array}{l}\text { University of British Columbia, Canada } \\
\text { ilaba@math.ubc.ca }\end{array}$ & Gilles Lebeau & $\begin{array}{l}\text { Université de Nice Sophia Antipolis, France } \\
\text { lebeau@unice.fr }\end{array}$ \\
\hline László Lempert & $\begin{array}{l}\text { Purdue University, USA } \\
\text { lempert@math.purdue.edu }\end{array}$ & Richard B. Melrose & $\begin{array}{l}\text { Massachussets Institute of Technology, USA } \\
\text { rbm@math.mit.edu }\end{array}$ \\
\hline Frank Merle & $\begin{array}{l}\text { Université de Cergy-Pontoise, France } \\
\text { Frank.Merle@u-cergy.fr }\end{array}$ & William Minicozzi II & $\begin{array}{l}\text { Johns Hopkins University, USA } \\
\text { minicozz@ math.jhu.edu }\end{array}$ \\
\hline Werner Müller & $\begin{array}{l}\text { Universität Bonn, Germany } \\
\text { mueller@math.uni-bonn.de }\end{array}$ & Yuval Peres & $\begin{array}{l}\text { University of California, Berkeley, USA } \\
\text { peres@stat.berkeley.edu }\end{array}$ \\
\hline Gilles Pisier & $\begin{array}{l}\text { Texas A\&M University, and Paris } 6 \\
\text { pisier@math.tamu.edu }\end{array}$ & Tristan Rivière & $\begin{array}{l}\text { ETH, Switzerland } \\
\text { riviere@math.ethz.ch }\end{array}$ \\
\hline Igor Rodnianski & $\begin{array}{l}\text { Princeton University, USA } \\
\text { irod@math.princeton.edu }\end{array}$ & Wilhelm Schlag & $\begin{array}{l}\text { University of Chicago, USA } \\
\text { schlag@math.uchicago.edu }\end{array}$ \\
\hline Sylvia Serfaty & $\begin{array}{l}\text { New York University, USA } \\
\text { serfaty@ cims.nyu.edu }\end{array}$ & Yum-Tong Siu & $\begin{array}{l}\text { Harvard University, USA } \\
\text { siu@math.harvard.edu }\end{array}$ \\
\hline Terence Tao & $\begin{array}{l}\text { University of California, Los Angeles, USA } \\
\text { tao@math.ucla.edu }\end{array}$ & Michael E. Taylor & $\begin{array}{l}\text { Univ. of North Carolina, Chapel Hill, USA } \\
\text { met@math.unc.edu }\end{array}$ \\
\hline Gunther Uhlmann & $\begin{array}{l}\text { University of Washington, USA } \\
\text { gunther@math.washington.edu }\end{array}$ & András Vasy & $\begin{array}{l}\text { Stanford University, USA } \\
\text { andras@math.stanford.edu }\end{array}$ \\
\hline an Virgil Voiculescu & $\begin{array}{l}\text { University of California, Berkeley, USA } \\
\text { dvv@ math.berkeley.edu }\end{array}$ & Steven Zelditch & $\begin{array}{l}\text { Johns Hopkins University, USA } \\
\text { szelditch@math.jhu.edu }\end{array}$ \\
\hline
\end{tabular}

\section{PRODUCTION}

apde@mathscipub.org

Silvio Levy, Scientific Editor

Sheila Newbery, Senior Production Editor

See inside back cover or pjm.math.berkeley.edu/apde for submission instructions.

The subscription price for 2011 is US \$120/year for the electronic version, and \$180/year for print and electronic. Subscriptions, requests for back issues from the last three years and changes of subscribers address should be sent to Mathematical Sciences Publishers, Department of Mathematics, University of California, Berkeley, CA 94720-3840, USA.

Analysis \& PDE, at Mathematical Sciences Publishers, Department of Mathematics, University of California, Berkeley, CA 94720-3840 is published continuously online. Periodical rate postage paid at Berkeley, CA 94704, and additional mailing offices.

APDE peer review and production are managed by EditFLOW ${ }^{\mathrm{TM}}$ from Mathematical Sciences Publishers.

PUBLISHED BY

mathematical sciences publishers

http://www.mathscipub.org

A NON-PROFIT CORPORATION

Typeset in IATEX

Copyright (C2011 by Mathematical Sciences Publishers 


\section{ANALYSIS \& PDE}

\section{Volume $4 \quad$ No. $1 \quad 2011$}

Strichartz estimates on asymptotically hyperbolic manifolds

JEAN-MARC BOUCLET

Asymptotic behaviors of nonvariational elliptic systems

SZU-YU SOPHIE CHEN

Global regularity for the Navier-Stokes equations with some classes of large initial data

MARIUS PAICU and ZHIFEI ZHANG

Dynamics on Grassmannians and resolvents of cone operators

Juan B. Gil, Thomas Krainer and Gerardo A. Mendoza

Three-term commutator estimates and the regularity of $\frac{1}{2}$-harmonic maps into spheres 